\title{
Internal feedback stabilization of multi-dimensional wave equations with a boundary delay: a numerical study
}

\author{
Kaïs Ammari ${ }^{1}$, Boumediène Chentouf ${ }^{2}$ and Nejib Smaoui ${ }^{2 *}$ (D)
}

${ }^{*}$ Correspondence:

n.smaoui@ku.edu.kw; nsmaoui64@yahoo.com

2 Department of Mathematics, Faculty of Science, Kuwait

University, Safat 13060, Kuwait Full list of author information is available at the end of the article

\begin{abstract}
In this paper, we consider two internal stabilization problems for the multi-dimensional wave equation with a boundary time-delay. We prove that the first problem is well-posed in an appropriate functional space. Subsequently, we numerically study the exponential stability in a two-dimensional case under Geometric Control Condition (GCC) derived by Lebeau. In addition, we provide a numerical investigation of the second wave system, which corresponds to the two-dimensional variant of the system studied by Datko et al.
\end{abstract}

MSC: 35B05; 93D15; 93D20

Keywords: Internal stabilization; Boundary delay; Wave equations

\section{Introduction}

This article is devoted to the internal stabilization of a wave equation in an open bounded domain $\Omega$ of $\mathbb{R}^{n}(n \geq 2)$, where a boundary time-delay occurs. In the sequel, we denote by $\Gamma$ the boundary of $\Omega$ and assume that it is smooth (e.g., of class $C^{2}$ ), and $\Gamma=\overline{\Gamma_{0}} \cup \overline{\Gamma_{1}}$, where $\Gamma_{0}, \Gamma_{1}$ are open subsets of $\Gamma$ with $\Gamma_{0} \cap \Gamma_{1}=\emptyset$. Moreover, we suppose that the measure of $\Gamma_{0}$ is positive. The problem under consideration is the following boundary time-delayed wave system:

$$
\begin{aligned}
& u_{t t}(x, t)-\Delta u(x, t)+a(x) u_{t}(x, t)=0, \quad x \in \Omega, t>0, \\
& u(x, t)=0, \quad x \in \Gamma_{0}, t>0, \\
& u(x, t)=-k \mathcal{U}\left(u_{t}(x, t-\tau)\right), \quad x \in \Gamma_{1}, t>0, \\
& u(x, 0)=u_{0}(x), \quad u_{t}(x, 0)=u_{1}(x), \quad x \in \Omega, \\
& u_{t}(x, t)=f(x, t), \quad x \in \Gamma_{1}, t \in(-\tau, 0),
\end{aligned}
$$

where $\mathcal{U}\left(u_{t}(x, t-\tau)\right)$ represents the boundary control involving the time-delayed velocity term $u_{t}(x, t-\tau),(x, t) \in \Gamma_{1} \times \mathbb{R}_{+}$, in which $\mathbb{R}_{+}=(0, \infty)$ and $\tau>0$ is the time delay. Finally,

(c) The Author(s) 2022. This article is licensed under a Creative Commons Attribution 4.0 International License, which permits use, sharing, adaptation, distribution and reproduction in any medium or format, as long as you give appropriate credit to the original author(s) and the source, provide a link to the Creative Commons licence, and indicate if changes were made. The images or other third party material in this article are included in the article's Creative Commons licence, unless indicated otherwise in a credit line to the material. If material is not included in the article's Creative Commons licence and your intended use is not permitted by statutory regulation or exceeds the permitted use, you will need to obtain permission directly from the copyright holder. To view a copy of this licence, visit http://creativecommons.org/licenses/by/4.0/. 
$k$ is a real number, $a(\cdot) \in L^{\infty}(\Omega)$ such that $a(x) \geq 0$, on $\Omega$, and the initial data are taken in appropriate spaces.

Delay effects arise in many applications and practical problems. In particular, time delays are ubiquitous in control systems since sensors and actuators are commonly used. Based on numerous articles, it has been noticed that even an arbitrarily small delay may destabilize a system which was uniformly asymptotically stable in the absence of a time-delay (see, e.g., [20, 21]). Therefore, it is crucial to investigate the effect of a delay either on the stability of the system or on the performance of the controller. Several articles appeared in this research avenue aiming to eliminate the delay or compensate it, or at least reduce its negative impact. In contrast to this predominant view, a number of unstable systems can be stabilized via a purposeful introduction of a time-delay in the systems [11, 24], while others are not affected by the presence of a delay [23].

Now, let us go back to the system under consideration (1.1)-(1.5). First, we consider the boundary control $\mathcal{U}\left(u_{t}(x, t-\tau)=\frac{\partial G u_{t}}{\partial v}(x, t-\tau),(x, t) \in \Gamma_{1} \times \mathbb{R}_{+}\right.$, where $v$ stands for the unit normal vector of $\Gamma$ pointing towards the exterior of $\Omega$, and $\frac{\partial}{\partial \nu}$ is the normal derivative and in which $G=(-\Delta)^{-1}: H^{-1}(\Omega) \longrightarrow H_{0}^{1}(\Omega)$. This gives rise to our first problem:

$$
\begin{aligned}
& u_{t t}(x, t)-\Delta u(x, t)+a(x) u_{t}(x, t)=0, \quad x \in \Omega, t>0, \\
& u(x, t)=0, \quad x \in \Gamma_{0}, t>0, \\
& u(x, t)=-k \frac{\partial G u_{t}}{\partial v}(x, t-\tau), \quad x \in \Gamma_{1}, t>0, \\
& u(x, 0)=u_{0}(x), \quad u_{t}(x, 0)=u_{1}(x), \quad x \in \Omega, \\
& u_{t}(x, t)=f(x, t), \quad x \in \Gamma_{1}, t \in(-\tau, 0) .
\end{aligned}
$$

The exponential stability of (1.6)-(1.10) with $k=0$ has been studied in [28], where it has been shown that the system is exponentially stable if the support of $a$ satisfies some geometric control condition (GCC). In turn, if $\tau=0$ and $k<0$, that is, in the absence of any time-delay, then the above system (1.1)-(1.5) is exponentially stable when the support of $a$ or $\Gamma_{1}$ satisfies a geometrical control condition (GCC) (see $\left.[9,10,27,28]\right)$ even if $k=0$ (see, e.g., [28] and [36]). Nevertheless, if a delay term occurs in the system, then instability phenomena may arise as shown in [31] for Neumann feedback boundary control.

In order to contrast the destabilizing effect of the boundary time delay in a wave equation, a "good" (undelayed) damping term is introduced in (1.6) (see [31]). More precisely, the problem considered in [31] is

$$
\begin{aligned}
& u_{t t}(x, t)-\Delta u(x, t)+a u_{t}(x, t-\tau)+a_{0} u_{t}(x, t)=0, \quad x \in \Omega, t>0, \\
& u(x, t)=0, \quad x \in \Gamma_{0}, t>0, \\
& \frac{\partial u}{\partial v}(x, t)=0, \quad x \in \Gamma_{1}, t>0, \\
& u(x, 0)=u_{0}(x), \quad u_{t}(x, 0)=u_{1}(x), \quad x \in \Omega, \\
& u_{t}(x, t)=f(x, t), \quad x \in \Omega, t \in(-\tau, 0),
\end{aligned}
$$


with $a_{0}, a>0$ are real constants, and the initial data belong to suitable spaces. If $a_{0}>a$, it was shown in [31] that system (1.11)-(1.15) is uniformly exponentially stable (see also $[1,3,4,6,7,12,22,32,33]$ for related results and similar situations).

In the present paper, we shall focus on the numerical study of the effect of the time-delay on the stability of the system (1.6)-(1.10) under the action of the internal feedback control $a(x) u_{t}(x, t)$, for $x \in \Omega$ and $t>0$. With regard to the second problem, we take $\mathcal{U}\left(u_{t}(x, t-\tau)\right)=$ $u_{t}(x, t-\tau),(x, t) \in \Gamma_{1} \times \mathbb{R}_{+}$in (1.1)-(1.5) and also provide a numerical investigation of the corresponding closed-loop system, namely,

$$
\begin{aligned}
& u_{t t}(x, t)-\Delta u(x, t)+a(x) u_{t}(x, t)=0, \quad x \in \Omega, t>0, \\
& u(x, t)=0, \quad x \in \Gamma_{0}, t>0, \\
& \frac{\partial u}{\partial v}(x, t)=-k u_{t}(x, t-\tau), \quad x \in \Gamma_{1}, t>0, \\
& u(x, 0)=u_{0}(x), \quad u_{t}(x, 0)=u_{1}(x), \quad x \in \Omega, \\
& u_{t}(x, t)=f(x, t), \quad x \in \Gamma_{1}, t \in(-\tau, 0) .
\end{aligned}
$$

It is worth mentioning that the input/output operator of the system (1.16)-(1.20) is not admissible, which means that the open-loop system (1.16)-(1.17), (1.19) with $\frac{\partial u}{\partial v}=g \in$ $L_{\mathrm{loc}}^{2}\left(0,+\infty ; L^{2}\left(\Gamma_{1}\right)\right)$, for $(x, t) \in \Gamma_{1} \times(0, \infty)$, and $u_{0}=u_{1}=0$ satisfies $u_{t \mid \Gamma_{1}} \notin L_{\mathrm{loc}}^{2}(0,+\infty$; $\left.L^{2}\left(\Gamma_{1}\right)\right)$ (see [34]). With regard to the system without delay, the reader is referred to [14$18,25,26,30]$.

On the other hand, we note that studying the system (1.16)-(1.20) is mainly motivated by the work [21], where one can find an interesting analysis conducted for the onedimensional case of the system (1.16)-(1.20). Indeed, a positive answer to the above problem (concern) is provided in [21]. Specifically, the authors in [21] considered the following one-dimensional system

$$
\begin{aligned}
& u_{t t}(x, t)-u_{x x}(x, t)+2 b u_{t}(x, t)+b^{2} u(x, t)=0, \quad 0<x<1, t>0, \\
& u(0, t)=0, \quad t>0, \\
& u_{x}(1, t)=-c u_{t}(1, t-\tau), \quad t>0, \\
& u(x, 0)=u_{0}(x), u_{t}(x, 0)=u_{1}(x), \quad x \in(0,1), \\
& u_{t}(1, t)=f(t), \quad t \in(-\tau, 0),
\end{aligned}
$$

where $b$ and $c$ are positive real numbers. Moreover, the initial data and the function $f$ are in appropriate functional spaces. Through careful spectral analysis, the authors have shown in [21] that, for any $a>0$, and if $c$ satisfies

$$
0<c<\frac{1-e^{-2 b}}{1+e^{-2 b}}
$$

then the spectrum of the system (1.21)-(1.23) satisfies the following property:

$\operatorname{Re}(\omega) \leq-\beta$ for each eigenvalue $\omega$ of the system and some positive constant $\beta$ depending on the delay $\tau$. Notwithstanding, as far as we know, there are no results in the literature 
for the multi-dimensional case described by (1.16)-(1.20). This has motivated us to numerically solve this problem in the case when the space variable $x$ belongs to $\mathbb{R}^{2}$.

Finally, we would like to point out that one variant of the one-dimensional version of the system (1.6)-(1.10), where $a=f=0$ and for $\tau=2$, has the following form:

$$
\begin{aligned}
& u_{t t}(x, t)-u_{x x}(x, t)=0, \quad 0<x<1, t>0, \\
& u(0, t)=0, \quad t>0, \\
& u(1, t)=0, \quad t \in(0,2), \\
& u(1, t)=-k\left[\left(-\partial_{x}^{2}\right)^{-1} u_{t}\right](1, t-2), \quad t>2, \\
& u(x, 0)=u_{0}(x), u_{t}(x, 0)=u_{1}(x), \quad x \in(0,1),
\end{aligned}
$$

where $k$ is a real number. Arguing as in [5] (see the appendix), one can obtain the exponential stability of the above system for $\left(u_{0}, u_{1}\right) \in L^{2}(0,1) \times H^{-1}(0,1)$ provided that $k \in(-1,0)$.

The paper is organized as follows: The second section is devoted to the well-posedness of the problem (1.6)-(1.10). In the third section, we numerically study in $\mathbb{R}^{2}$ the exponential stability of both delayed systems (1.6)-(1.10) and (1.16)-(1.20). A short discussion is also provided in an appendix about the one-dimensional case of (1.6)-(1.10), namely, (1.27)-(1.31).

\section{Well-posedness of the problem (1.6)-(1.10)}

Let $A=-\Delta$ be the unbounded operator in $H=H^{-1}(\Omega)$ with domain

$$
H_{1}=\mathcal{D}(A)=H_{0}^{1}(\Omega)
$$

Next, we define a bounded linear operator $B \in \mathcal{L}\left(H^{-1}(\Omega)\right)$ as follows:

$$
B u=B^{*} u=\sqrt{a} u, \quad \forall u \in H^{-1}(\Omega) .
$$

Then, let $C \in \mathcal{L}\left(L^{2}\left(\Gamma_{1}\right) ; H_{-\frac{1}{2}}\right)$ such that

$$
C u=A_{-1} D u, \quad \forall u \in L^{2}\left(\Gamma_{1}\right), \quad C^{*} w=\left.\frac{\partial G w}{\partial v}\right|_{\Gamma_{1}}, \quad \forall w \in \mathcal{D}\left(A^{\frac{1}{2}}\right)=H_{\frac{1}{2}}=L^{2}(\Omega)
$$

where $H_{-\frac{1}{2}}=\left(\mathcal{D}\left(A^{\frac{1}{2}}\right)\right)^{\prime}$ (the duality is in the sense of $H$ ), while $A_{-1}$ is the extension of $A$ to $H$, namely, for all $h \in H$ and $\varphi \in \mathcal{D}(A), A_{-1} h$ is the unique element in $H_{-1}=(\mathcal{D}(A))^{\prime}$ (the duality is in the sense of $H$ ) such that (see, for instance, [35])

$$
\left\langle A_{-1} h, \varphi\right\rangle_{H_{-1}, H_{1}}=\langle h, A \varphi\rangle_{H}
$$

Here and in the sequel, $D \in \mathcal{L}\left(L^{2}\left(\Gamma_{1}\right) ; L^{2}(\Omega)\right)$ (called the Dirichlet mapping), $\forall v \in L^{2}\left(\Gamma_{1}\right)$, $D v$ is the unique solution (transposition solution) of

$$
\Delta D v=0, \quad D v_{\mid \Gamma_{0}}=0, \quad D v_{\mid \Gamma_{1}}=v .
$$


To study the well-posedness of the systems (1.6)-(1.10), we write it as an abstract Cauchy problem in a product space and use the semigroup approach. For this purpose, take the Hilbert space $\mathcal{H}:=H_{\frac{1}{2}} \times H$ and the unbounded linear operators

$$
\mathcal{A}: \mathcal{D}(\mathcal{A})=H_{1} \times H_{\frac{1}{2}} \subset \mathcal{H} \longrightarrow \mathcal{H}, \quad \mathcal{A}\left(\begin{array}{l}
u_{1} \\
u_{2}
\end{array}\right)=\left(\begin{array}{c}
u_{2} \\
-A u_{1}-B B^{*} u_{2}
\end{array}\right) .
$$

It is well known that the operator $(\mathcal{A}, \mathcal{D}(\mathcal{A}))$ defined by $(2.1)$, generates a strongly continuous semigroup of contractions on $\mathcal{H}$ denoted by $(\mathcal{T}(t))_{t \geq 0}$. We also denote $\left(\mathcal{T}_{-1}(t)\right)_{t \geq 0}$ the extension of $(\mathcal{T}(t))_{t \geq 0}$ to $(\mathcal{D}(\mathcal{A}))^{\prime}:=H \times H_{-\frac{1}{2}}$, where $H_{-\frac{1}{2}}=\left(\mathcal{D}\left(A^{\frac{1}{2}}\right)\right)^{\prime}$, and the duality is in the sense of $H$.

We have the following result:

Theorem 2.1 The system (1.6)-(1.10) is well-posed. More precisely, for every $\left(u_{0}, u_{1}\right) \in \mathcal{H}$, and $f \in L^{2}\left(-\tau, 0 ; L^{2}\left(\Gamma_{1}\right)\right)$, the solution of $(1.6)-(1.10)$ is given by

$$
\left(\begin{array}{c}
u(t) \\
u_{t}(t)
\end{array}\right)=\left\{\begin{aligned}
\left(\begin{array}{c}
u^{0}(t) \\
u_{t}^{0}(t)
\end{array}\right)= & \mathcal{T}(t)\left(\begin{array}{l}
u_{0} \\
u_{1}
\end{array}\right)-\int_{0}^{t} \mathcal{T}_{-1}(t-s)\left(\begin{array}{c}
0 \\
k C f(s-\tau)
\end{array}\right) d s, \quad t \in[0, \tau], \\
\left(\begin{array}{c}
u^{j}(t) \\
u_{t}^{j}(t)
\end{array}\right)= & \mathcal{T}(t-j \tau)\left(\begin{array}{l}
w^{j-1}(j \tau) \\
u_{t}^{j-1}(j \tau)
\end{array}\right) \\
& -\int_{j \tau}^{t} \mathcal{T}_{-1}(t-s)\left(\begin{array}{l}
k C C^{*} u_{t}^{j-1}(s-\tau) \\
0
\end{array}\right) d s, \quad t \in[j \tau,(j+1) \tau], j \geq 1
\end{aligned}\right.
$$

and satisfies $\left(u^{j}, u_{t}^{j}\right) \in C([j \tau,(j+1) \tau], \mathcal{H}), j \in \mathbb{N}$.

The proof requires several steps (see $[6,8]$ for other types of problems). First, consider the evolution problems

$$
\begin{aligned}
& y_{t t}^{j}(t)+A y^{j}(t)+B B^{*} y_{t}^{j}(t)=k C v^{j}(t), \quad \text { in }(j \tau,(j+1) \tau), j \in \mathbb{N}^{*}, \\
& y^{j}(j \tau)=y_{t}^{j}(j \tau)=0, \quad j \in \mathbb{N}^{*} .
\end{aligned}
$$

and

$$
\begin{aligned}
& \phi_{t t}(t)+A \phi(t)+B B^{*} \phi_{t}(t)=0, \quad \text { in }(0,+\infty), \\
& \phi(0)=\phi_{0}, \quad \phi_{t}(0)=\phi_{1} .
\end{aligned}
$$

A natural question is the regularity of $y^{j}$ when $v^{j} \in L^{2}\left(\tau j,(j+1) \tau ; L^{2}\left(\Gamma_{1}\right)\right), j \in \mathbb{N}^{*}$. By applying standard energy estimates, we can easily check that $y^{j} \in C([j \tau,(j+1) \tau] ; H) \cap C^{1}([j \tau,(j+$ $\left.1) \tau] ; H_{-\frac{1}{2}}\right)$. However, if $C$ satisfies a certain admissibility condition, then $y^{j}$ is more regular. More precisely, the following result, which is a version of the general transposition method (see, for instance, Lions and Magenes [29]) holds true: The system (2.4)-(2.5) admits a unique solution $\phi$ having the regularity

$$
\begin{aligned}
& \phi \in C\left([0, \tau] ; H_{\frac{1}{2}}\right) \cap C^{1}([0, \tau] ; H), \quad \forall\left(\phi_{0}, \phi_{1}\right) \in \mathcal{H}, \\
& \left(\phi, \phi_{t}\right)^{t}(t)=\mathcal{T}(t)\left(\begin{array}{c}
\phi_{0} \\
\phi_{1}
\end{array}\right), \quad 0 \leq t \leq \tau .
\end{aligned}
$$


Moreover, according to [2], $C^{*} \phi(\cdot) \in H^{1}\left(0, \tau ; L^{2}\left(\Gamma_{1}\right)\right)$ and for all $t \in(0, \tau)$, there exists a constant $C>0$ such that

$$
\left\|\left(C^{*} \phi\right)^{\prime}(\cdot)\right\|_{L^{2}\left(0, T ; L^{2}\left(\Gamma_{1}\right)\right)} \leq C\left\|\left(\phi_{0}, \phi_{1}\right)\right\|_{\mathcal{H}^{\prime}}, \quad \forall\left(\phi_{0}, \phi_{1}\right) \in \mathcal{H} .
$$

Next, we have the following lemma:

Lemma 2.2 Suppose that $v^{j} \in L^{2}\left([j \tau,(j+1) \tau] ; L^{2}\left(\Gamma_{1}\right)\right), j \in \mathbb{N}^{*}$. Then the problem $(2.2)-(2.3)$ admits a unique solution having the regularity

$$
y^{j} \in C\left([j \tau,(j+1) \tau] ; H_{\frac{1}{2}}\right) \cap C^{1}([j \tau,(j+1) \tau] ; H), \quad j \in \mathbb{N}^{*},
$$

and

$$
\left(y^{j}, y_{t}^{j}\right)^{t}(t)=\int_{j \tau}^{t} \mathcal{T}_{-1}(t-s)\left(\begin{array}{c}
0 \\
k C v_{j}(s)
\end{array}\right) d s, \quad j \tau \leq t \leq(j+1) \tau, j \geq 1
$$

Proof Setting $Z(t)=\left(\begin{array}{c}y^{j}(t+j \tau) \\ y_{t}^{j}(t+j \tau)\end{array}\right)$, the system (2.2)-(2.3) can be written as

$$
Z_{t}^{j}=\mathcal{A} Z^{j}(t)+k \mathcal{C} v^{j}(t+j \tau) \quad \text { on }(0, \tau), \quad Z^{j}(0)=0,
$$

where

$$
\mathcal{A}=\left(\begin{array}{cc}
0 & I \\
-A-B B^{*}
\end{array}\right): \mathcal{H} \rightarrow[\mathcal{D}(\mathcal{A})]^{\prime}, \quad \mathcal{C}=\left(\begin{array}{l}
0 \\
C
\end{array}\right): L^{2}\left(\Gamma_{1}\right) \rightarrow[\mathcal{D}(\mathcal{A})]^{\prime} .
$$

After simple calculations, one can check that the operator $\mathcal{C}^{*}: \mathcal{D}(\mathcal{A}) \rightarrow L^{2}\left(\Gamma_{1}\right)$ is given by

$$
\mathcal{C}^{*}\left(\begin{array}{l}
u^{j} \\
v^{j}
\end{array}\right)=C^{*} v^{j}, \quad \forall\left(u^{j}, v^{j}\right) \in \mathcal{D}(\mathcal{A})
$$

This implies that

$$
\mathcal{C}^{*} \mathcal{T}(t)\left(\begin{array}{c}
\phi_{0} \\
\phi_{1}
\end{array}\right)=C^{*} \phi_{t}(t), \quad \forall\left(\phi_{0}, \phi_{1}\right) \in \mathcal{D}(\mathcal{A})
$$

in which $\phi$ satisfies (2.4)-(2.5). Amalgamating the last claim with (2.6), we deduce that there exists a constant $C>0$ such that for all $T \in(0, \tau)$

$$
\int_{0}^{T} \int_{\Gamma_{1}}\left|\mathcal{C}^{*} \mathcal{T}(t)\left(\begin{array}{c}
\phi_{0} \\
\phi_{1}
\end{array}\right)\right|^{2} d \Gamma_{1} d t \leq C\left\|\left(\phi_{0}, \phi_{1}\right)\right\|_{\mathcal{H}}^{2}, \quad \forall\left(\phi_{0}, \phi_{1}\right) \in \mathcal{D}(\mathcal{A})
$$

In view of [13, Theorem 3.1, p.187], the last estimate leads to the interior regularity (2.7) (see also [35]). 
The existence result for problem (1.6)-(1.10) can now be established by induction. In fact, we first set on $[0, \tau]$ (case $j=0$ )

$$
\left(\begin{array}{l}
u^{0}(t) \\
u_{t}^{0}(t)
\end{array}\right)=\mathcal{T}(t)\left(\begin{array}{l}
u_{0} \\
u_{1}
\end{array}\right)-\int_{0}^{t} \mathcal{T}_{-1}(t-s)\left(\begin{array}{c}
0 \\
k C f(s-\tau)
\end{array}\right) d s, \quad \forall t \in[0, \tau] .
$$

The latter clearly provides a solution of $(1.6)-(1.9)$ on $(0, \tau)$. Moreover, such a solution has the regularity $\left(u^{0}, u_{t}^{0}\right) \in C([0, \tau] ; \mathcal{H})$. The next step is to consider $j \geq 1$ and then let for all $t \in[j \tau,(j+1) \tau]$,

$$
\begin{aligned}
\left(\begin{array}{c}
u^{j}(t) \\
u_{t}^{j}(t)
\end{array}\right) & =\left(\begin{array}{c}
\phi(t+j \tau) \\
\phi_{t}(t+j \tau)
\end{array}\right)+\left(\begin{array}{l}
y^{j}(t) \\
y_{t}^{j}(t)
\end{array}\right) \\
& =\mathcal{T}(t+j \tau)\left(\begin{array}{c}
u^{j-1}(j \tau) \\
u_{t}^{j-1}(j \tau)
\end{array}\right)-\int_{j \tau}^{t} \mathcal{T}_{-1}(t-s)\left(\begin{array}{c}
0 \\
k C C^{*} u_{t}^{j-1}(s-\tau)
\end{array}\right) d s,
\end{aligned}
$$

where $y^{j}$ (respectively $\phi$ ) is solution of (2.2)-(2.3) (respectively (2.4)-(2.5)) with $v^{j}(t)=$ $-C^{*} u_{t}^{j-1}(t-\tau)$ (that belongs to $L^{2}\left(j \tau,(j+1) \tau ; L^{2}\left(\Gamma_{1}\right)\right)$ because the input/output operator $C^{*}$ is admissible (see [2]) and $\phi_{0}=u^{j-1}(j \tau), \phi_{1}=u_{t}^{j-1}(j \tau)$. This solution has the announced regularity due to the above arguments.

\section{Numerical study}

Numerical solutions for the multi-dimensional wave systems (1.6) -(1.10) as well as (1.16) (1.20) with and without the presence of a boundary time-delay are simulated using COMSOL Multiphysics software. The domain $\Omega$ is taken to be the square $(0,1) \times(0,1)$ (see Fig. 1), while different values of $k$ and various functions $a(x), x=\left(x_{1}, x_{2}\right)^{T}$ are considered. It should also be noted that for the numerical experiments we assume that $\bar{\Gamma}_{0} \cap \bar{\Gamma}_{1} \neq \emptyset$, which is different from the assumption in the theoretical discussion.

The finite element method with the quadratic Lagrange shape functions as basis functions is used with a fixed fine element mesh size, where the minimum element mesh size equals to $1.25 \times 10^{-4}$, and the maximum element mesh size is 0.037 , and the generalized- $\alpha$ method as the time-stepping scheme is used with $d t=0.001$ (see Fig. 1). The generalized$\alpha$ method was introduced by Chung and Hulbert [19] to solve the following second-order ODEs:

$$
\begin{aligned}
& A \ddot{Y}(t)+B \dot{Y}(t)+C Y(t)=H(t), \\
& Y(0)=d \\
& \dot{Y}(0)=v
\end{aligned}
$$

where $A, B$, and $C$ are the mass, damping, and stiffness matrices, respectively, $H(t)$ is the vector applied loads, and $Y(t)$ is the displacement vector, where the superimposed dots indicate differentiation with respect to time; $d$ and $v$ are given vectors of initial displacements and velocities, respectively.

By approximating $Y\left(t_{i}\right), \dot{Y}\left(t_{i}\right)$, and $\ddot{Y}\left(t_{i}\right)$ by $d_{i}, v_{i}$, and $a_{i}$, respectively, the generalized- $\alpha$ algorithm is given by:

$$
d_{i+1}=d_{i}+\Delta t v_{i}+\Delta t^{2}\left(\left(\frac{1}{2}-\beta\right) a_{i}+\beta a_{i+1}\right),
$$




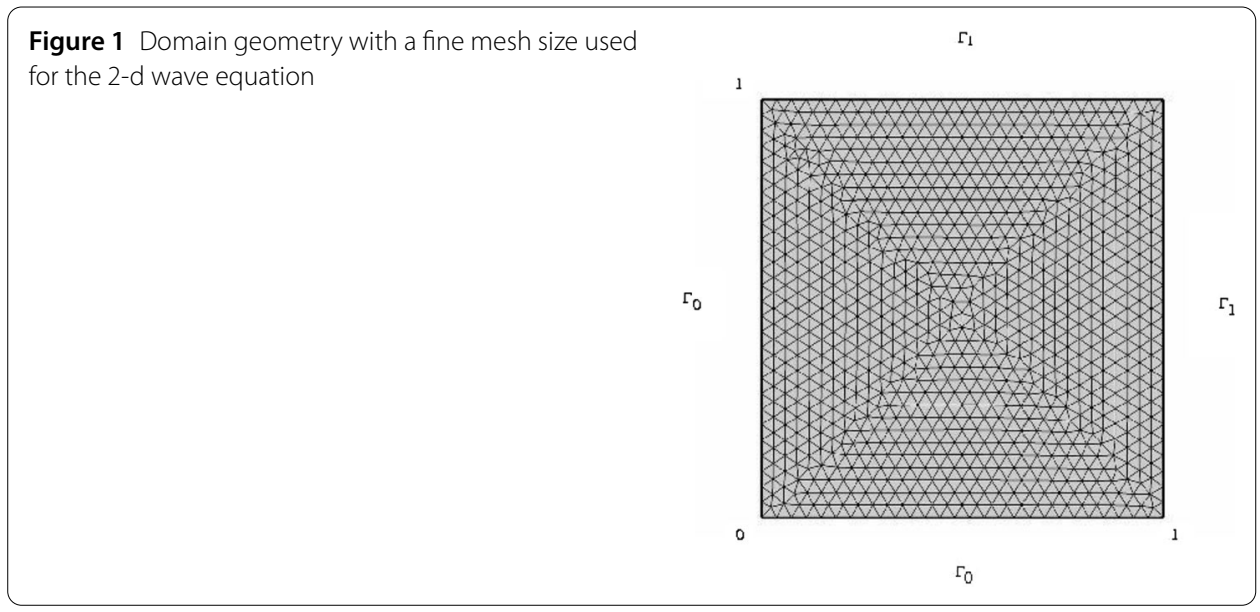

$$
\begin{aligned}
& v_{i+1}=v_{i}+\Delta t\left((1-\gamma) a_{i}+\gamma a_{i+1}\right), \\
& A a_{i+1-\alpha_{m}}+B v_{i+1-\alpha_{f}}+C d_{i+1-\alpha_{f}}=H\left(t_{i+1-\alpha_{f}}\right), \\
& d_{0}=d, \quad v_{0}=v, \quad a_{0}=A^{-1}(H(0)-B v-C d),
\end{aligned}
$$

where

$$
\begin{aligned}
& d_{i+1-\alpha_{f}}=\left(1-\alpha_{f}\right) d_{i+1}+\alpha_{f} d_{i}, \\
& v_{i+1-\alpha_{f}}=\left(1-\alpha_{f}\right) v_{i+1}+\alpha_{f} v_{i}, \\
& a_{i+1-\alpha_{m}}=\left(1-\alpha_{m}\right) a_{i+1}+\alpha_{m} a_{i}, \\
& t_{i+1-\alpha_{f}}=\left(1-\alpha_{f}\right) t_{i+1}+\alpha_{f} t_{i},
\end{aligned}
$$

in which $i \in[0, \ldots, N-1], N$ is the number of time steps, and $\Delta t=t_{i+1}-t_{i}$ is the time step size. The algorithm parameters $\alpha_{f}, \alpha_{m}, \beta$, and $\gamma$ are used to control the degree of damping high frequencies while minimizing unwanted low-frequency dissipation. The values of the parameters $\alpha_{f}, \alpha_{m}, \beta$, and $\gamma$ depend on the user-specified high-frequency dissipation $\rho_{\infty}$. According to [19], when $\alpha_{f}=\frac{\rho_{\infty}}{\rho_{\infty}+1} ; \alpha_{m}=\frac{2 \rho_{\infty}-1}{\rho_{\infty}+1} ; \beta=\frac{1}{4}\left(1-\alpha_{m}+\alpha_{f}\right)^{2}$, and $\gamma=\frac{1}{2}-\alpha_{m}+\alpha_{f}$, the algorithm is unconditionally stable and second-order more accurate as compared to other time-stepping schemes, especially for systems with the second-order derivatives. It should be noted that $\rho_{\infty}$ was taken to be 0.75 in all numerical simulations conducted herein. Therefore, the values of the parameters $\alpha_{f}, \alpha_{m}, \beta$, and $\gamma$ are $0.428,0.285,0.326$, and 0.642 , respectively.

\subsection{The system (1.6)-(1.10)}

For each regular solution of the system (1.6)-(1.10) in $L^{2}(\Omega) \times H^{-1}(\Omega)$, we consider the following functional

$$
E_{0}(t):=\frac{1}{2}\|u(t)\|_{L^{2}(\Omega)}^{2}
$$


The latter represents the energy of the displacement term $u(x, t)$ for $(1.6)-(1.10)$ as the energy is

$$
\frac{1}{2}\left\{\|u(t)\|_{L^{2}(\Omega)}^{2}+\left\|u_{t}(t)\right\|_{H^{-1}(\Omega)}^{2}\right\} .
$$

\subsubsection{The 2-d wave system without a boundary time-delay}

In this subsection, we consider the 2-d wave problem (1.6)-(1.10) without delay. Letting $x=\left(x_{1}, x_{2}\right)^{T}$ and taking the initial data $u_{0}(x)=0$ and $u_{1}(x)=\sin \left(\pi x_{1}\right)$, the system is:

$$
\begin{aligned}
& u_{t t}(x, t)-\Delta u(x, t)+a(x) u_{t}(x, t)=0, \quad x \in \Omega, t>0, \\
& u(x, t)=0, \quad x \in \Gamma_{0}, t>0, \\
& u(x, t)=-k \frac{\partial G u_{t}}{\partial v}(x, t), \quad x \in \Gamma_{1}, t>0, \\
& u(x, 0)=0, \quad u_{t}(x, 0)=\sin \left(\pi x_{1}\right), \quad x \in \Omega .
\end{aligned}
$$

First, we choose $a(x)=1$ and vary the values of $k$. It has been observed that the dynamics of the system (3.13)-(3.16) is exponentially stable if $k \leq 0$ and is unstable for $k>0$. Figure 2 is a $2-\mathrm{d}$ solution of $u(x, t)$ at it evolves in time for $k=1$, and Fig. 3a presents the

(a)

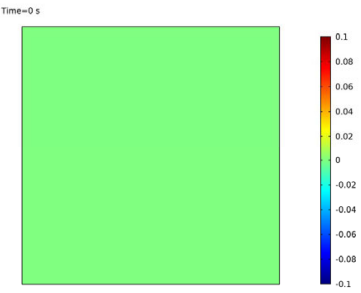

(d)

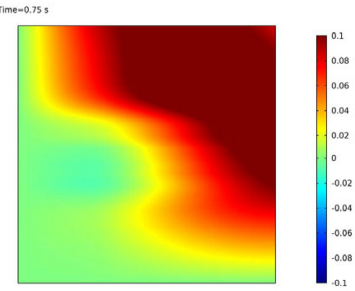

$(\mathrm{g})$

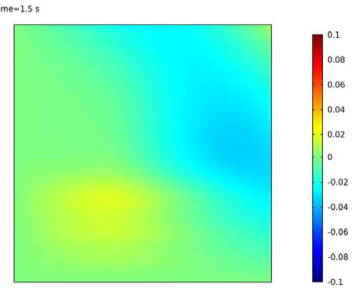

(b)

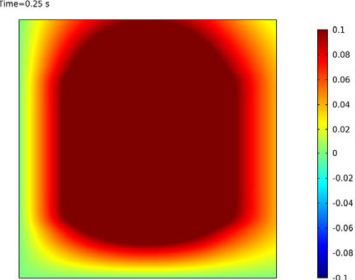

(e)

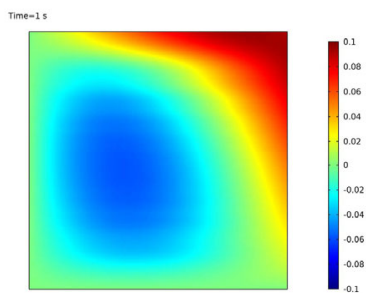

(h)

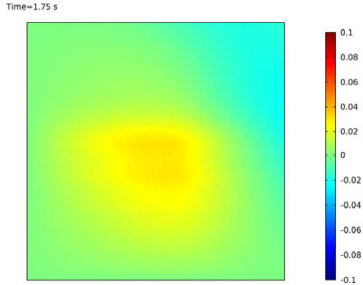

(c)

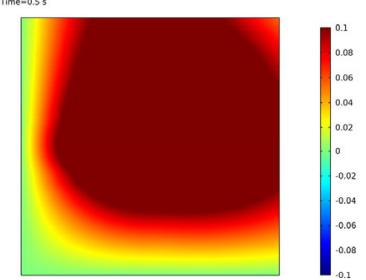

(f)

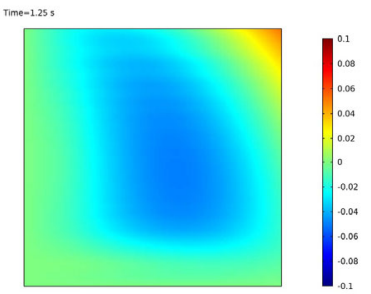

(i)

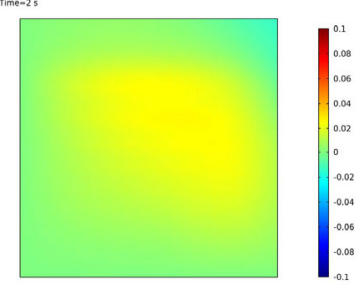

Figure 2 2-d solutions of the wave system without delay (3.13)-(3.16), when $a(x)=1$ and $k=-1$, at different times: $t=0, \ldots, 2$ 
(a)

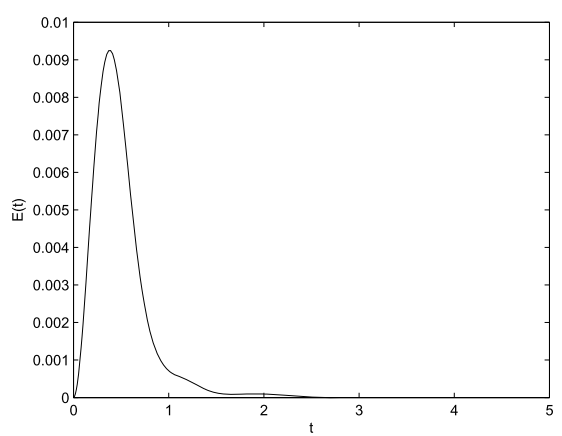

(b)

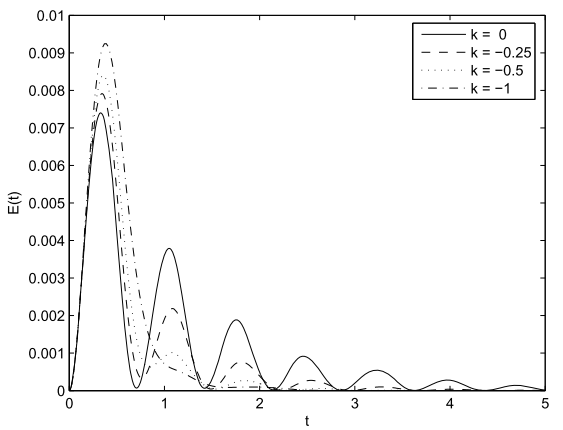

(c)

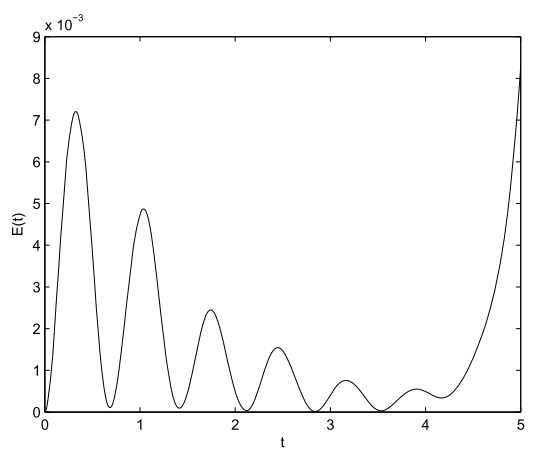

Figure 3 The energy $E_{0}(t)$ of the wave system without delay (3.13)-(3.16) when: a) $a(x)=1$, and $k=-1$; b) $a(x)=1$, and for negative values of $k ; c) a(x)=1$, and $k=0.1$

corresponding energy, $E_{0}(t)$, as defined by (3.12) versus time. It is clear that the dynamics of the system for this case exponentially converges to the zero solution. Figure $3 \mathrm{~b}$ is a plot of the energy $E_{0}(t)$ for different negative values of $k$, indicating that the energy exponentially converges faster as the value of $k$ decreases. On the other hand, Fig. $3 \mathrm{c}$ shows that the dynamics of the system is unstable when $k=0.1$.

\subsubsection{The 2-d wave equation (1.6)-(1.10) with a boundary time-delay}

In this subsection, we go back to the 2-d wave equation (1.6)-(1.10) with a boundary time-delay $\tau$. The inial data are taken as follows: $u_{0}(x)=0, u_{1}(x)=\sin \left(\pi x_{1}\right)$, and $f(x, t)=\sin \left(\pi x_{1}\right)$. The system is simulated for different parameters $k$, functions $a(x)$, and time-delays $\tau$. First, we choose the same value of $k=-1$ and function $a(x)=1$ as in the case without delay presented in Sect. 3.1.1, but with time-delay $\tau=1$. Figure 4 presents the dynamics of $u(x, t)$ at different time, and Fig. 5 shows the corresponding energy, $E_{0}(t)$, versus time. Figures 4-5 indicate that the presence of a time-delay destabilizes the dynamics of the originally exponentially stable system (compare with Figs. 2 and 3a). Moreover, choosing the function $a(x)=20$ and keeping the value of $k=-1$ with a time-delay $\tau=1$ exponentially stabilize the dynamics of the 2 -d wave equation to the zero solution (see Figs. 6 and 7a). It is worth mentioning that based on the above discussion, the choice of $a(x)$ plays an important role in the stability of the solution for the system (1.6)-(1.10) with a time-delay. 
(a)

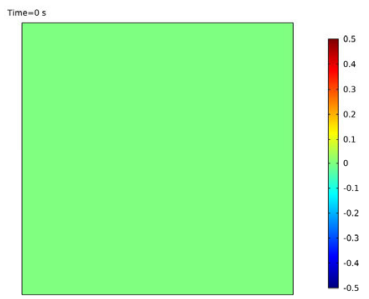

(d)

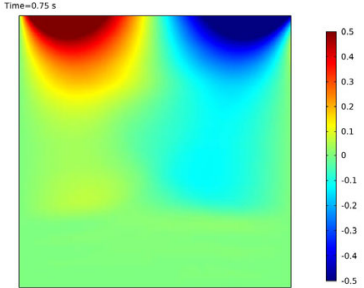

(g)

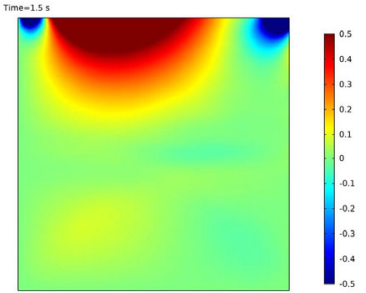

(b)

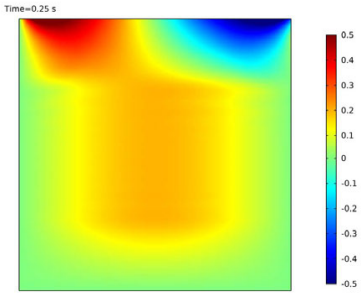

(e)

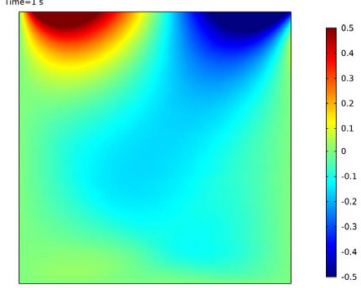

(h)

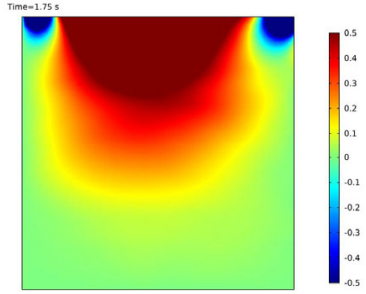

(c)

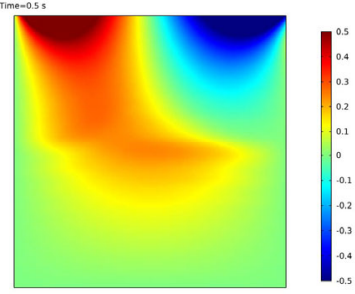

(f)

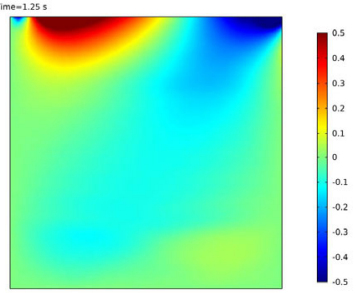

(i)

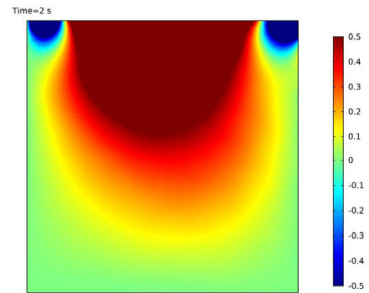

Figure 42 -d solutions of the wave equation (1.6)-(1.10) with a time-delay $\tau=1$ when $a(x)=1$ and $k=-1$, at different times: $t=0, \ldots, 2$

Figure 5 The energy $E_{0}(t)$ of the system (1.6)-(1.10) with a delay $\tau=1$ when $a(x)=1$, and $k=-1$

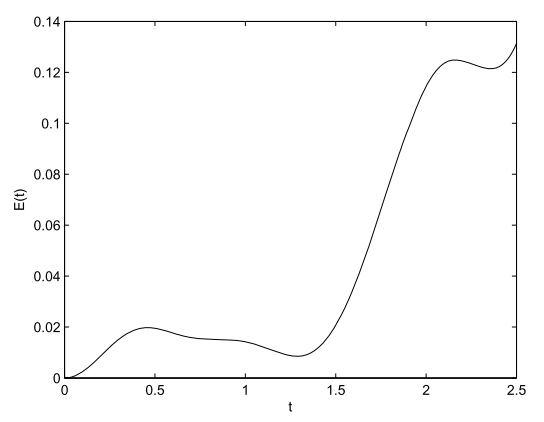

In turn, fixing $a(x)=1$ and a time-delay $\tau=1$ and choosing different values of $k$ change the dynamics of the 2-d wave equation (1.6)-(1.10) depending on the value of $k$. Figure 7b shows that the dynamics stabilizes as the value of $k$ increases from -0.2 to -0.1 . On the other hand, if the values of $k$ increase from 0.1 to 0.2 , the dynamics becomes more unstable (see Fig. 7c). These observations indicate that if $a(x)=1$ and $|k|<0.2$, then the 2 -d wave equation with a boundary time-delay $\tau=1$ is stable. However, this is not true if we change the function $a(x)=3$, where it is shown that the dynamics becomes exponentially stable (see Fig. 7d). Figure 7e indicates similar observations to Fig. $7 d$ when the time-delay is 
(a)

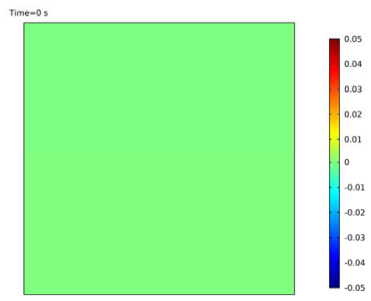

(d)

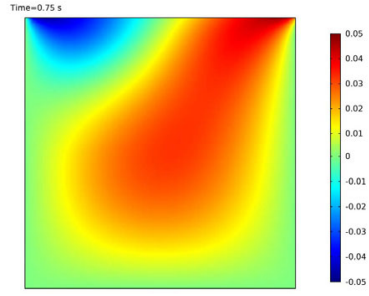

$(\mathrm{g})$

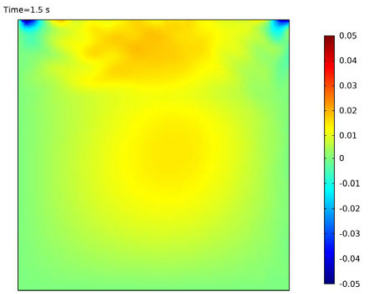

(b)

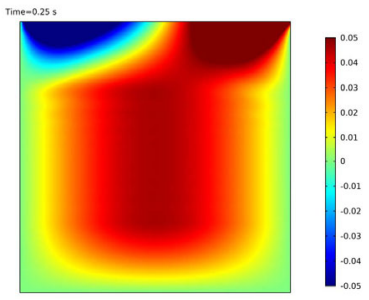

(e)

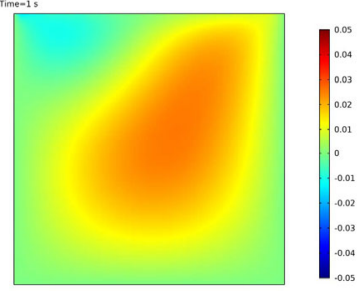

(h)

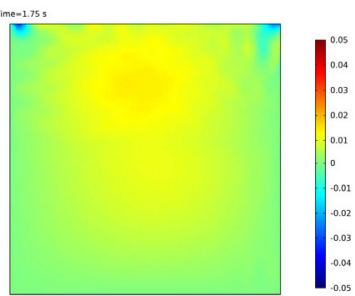

(c)

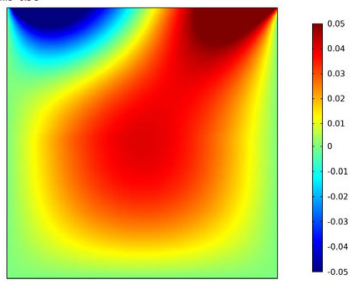

(f)

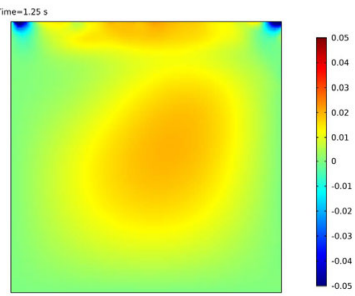

(i)

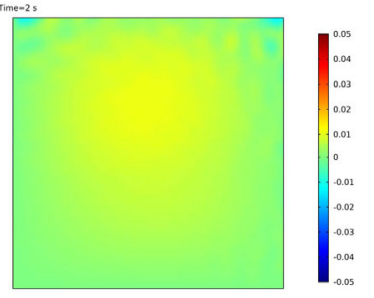

Figure 62 -d solutions of the wave equation (1.6)-(1.10) with a delay $\tau=1$ when $a(x)=20$ and $k=1$, at different times: $t=0, \ldots, 2$

increased to $\tau=2$. In addition, fixing $a(x)=0$ and $\tau=3$ and choosing different values of $k$ change the dynamics of the 2-d wave equation (1.6)-(1.10) depending on the value of $k$. Figures $7 \mathrm{f}$ and $7 \mathrm{~g}$ show that the system becomes more unstable as $|k|$ increases from 0.1 to 0.2 .

Now, to investigate the effect of the choice of the time-delay $\tau$ on the stability of the system (1.6)-(1.10), we fix $k$ and $a(x)$ and vary $\tau$. Figure 8 a shows that as we increase the value of the time-delay $\tau$ while fixing $k=-0.2$ and $a(x)=1$, the system destabilizes, and the rate of divergence of the energy increases as the time-delay $\tau$ increases. Similar observations are deduced for the case $k=0.2$ and $a(x)=1$ (see Fig. 8b). However, increasing the value of $a(x)=3$ exponentially stabilizes the dynamics for the previous two cases: $k=-0.2$, and $k=0.2$ (see Figs. $8 \mathrm{c}$ and $8 \mathrm{~d}$ ).

Next, we investigate the stability of the system (1.6)-(1.10) for different values of $\tau$ when the support $\Omega_{1}$ of the function $a$ satisfies a geometric control condition (GCC) (i.e., $\left.\Omega_{1}=\left(\frac{1}{3}, \frac{2}{3}\right) \mathrm{x}(0,1)\right)$. In addition, we fix $k$ and choose $a(x)=e^{\left(x_{1} x_{2}\right)}$, where $\left(x_{1}, x_{2}\right) \in \Omega_{1}$. Figure $9 \mathrm{a}$ depicts the energy, $E_{0}(t)$, versus time when $k=0.1$. Figure $9 \mathrm{a}$ indicates that as the time delay increases, the dynamics destabilizes. On the other hand, the dynamics stabilizes when $k=-0.1$ as the time delay increases (see Fig. 9b). The same observations are deduced for the case $k=0.2$ and $k=-0.2$ (see Figs. 9c and 9d, respectively). 
(a)

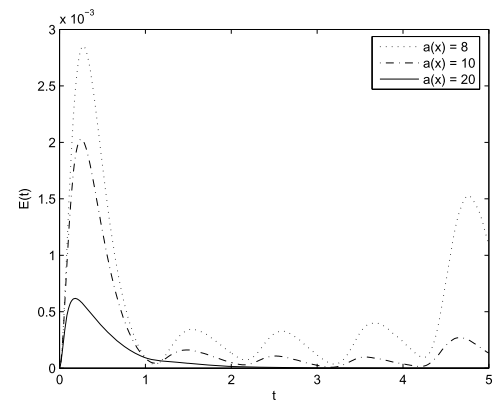

(c)

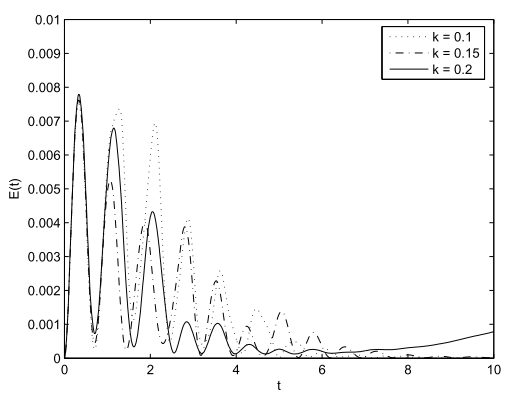

(e)

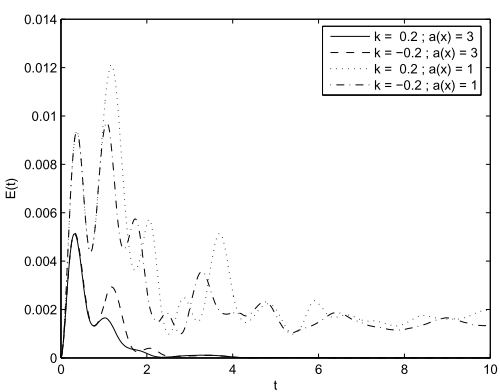

(b)

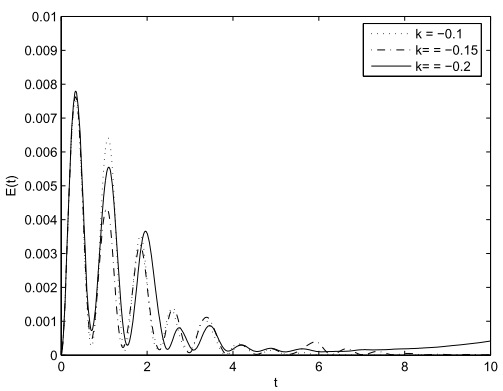

(d)

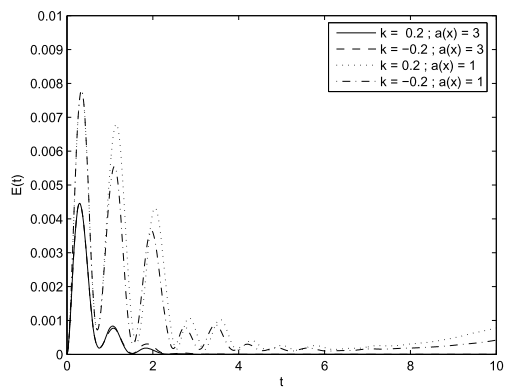

(f)

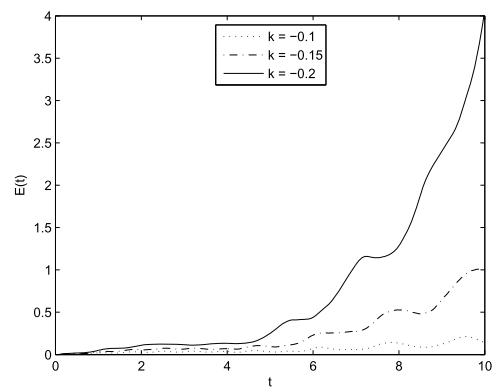

g)

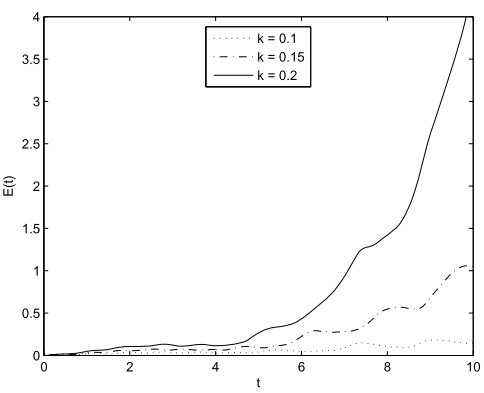

Figure 7 The energy $E_{0}(t)$ of the wave system (1.6)-(1.10) with: a) $\tau=1$ when $k=-1$, and for various $a(x)$; $b$ ) $\tau=1, k$ is negative and when $a(x)=1 ; c) \tau=1, k$ is positive and when $a(x)=1 ; d) \tau=1, a(x)=1$ and $a(x)=3$, and for various $k$; e) $\tau=2, a(x)=1$ and $a(x)=3$, and for different values of $k ; f) \tau=3, a(x)=0$, and for several values of $k ; g) \tau=3, a(x)=0$, and for $k>0$ 
(a)

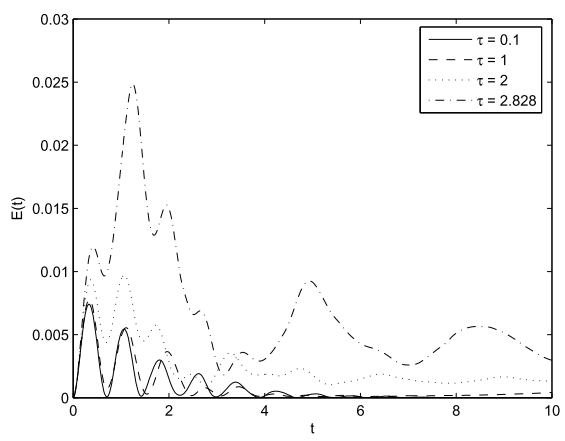

(c)

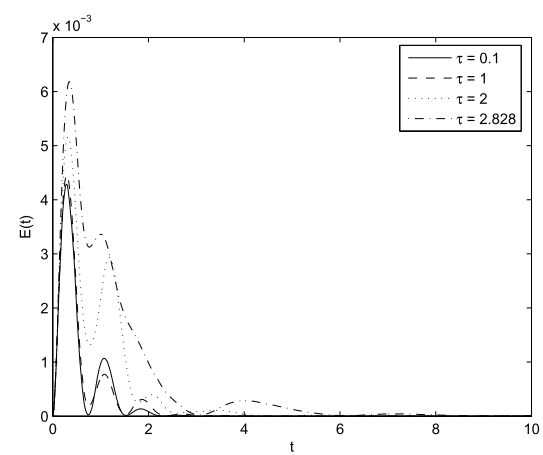

(b)

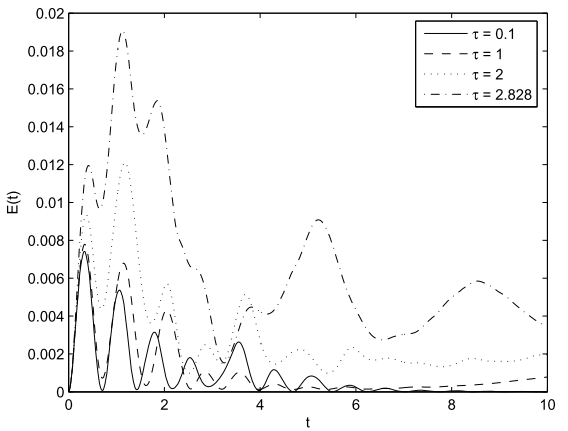

(d)

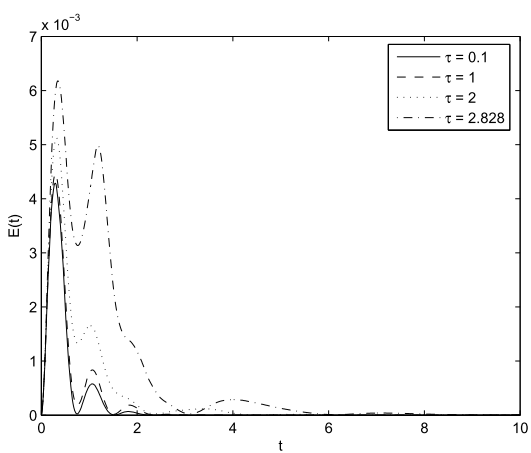

Figure 8 The energy $E_{0}(t)$ of the wave system (1.6)-(1.10) with: a) various delays when $a(x)=1$ and for $k=-0.2 ; b)$ different delays when $a(x)=1$ and for $k=0.2 ; c)$ several delays when $a(x)=3$ and for $k=-0.2 ; d$ ) various delays when $a(x)=3$ and for $k=0.2$

\subsection{The system (1.16)-(1.20)}

For each regular solution of the system (1.16)-(1.20), we consider the following functional as the total energy of the system

$$
E(t):=\frac{1}{2} \int_{\Omega}\left\{|\nabla u(x, t)|^{2}+\left|u_{t}(x, t)\right|^{2}\right\} d x
$$

\subsubsection{The 2-d wave system (1.16)-(1.20) without a boundary time-delay}

In this subsection, we consider the following 2-d wave system (1.16)-(1.20) but without the presence of the boundary delay:

$$
\begin{aligned}
& u_{t t}(x, t)-\Delta u(x, t)+a(x) u_{t}(x, t)=0, \quad x \in \Omega, t>0, \\
& u(x, t)=0, \quad x \in \Gamma_{0}, t>0, \\
& \frac{\partial u}{\partial v}(x, t)=-k u_{t}(x, t), \quad x \in \Gamma_{1}, t>0, \\
& u(x, 0)=u_{0}(x), \quad u_{t}(x, 0)=u_{1}(x), \quad x \in \Omega,
\end{aligned}
$$

where $x=\left(x_{1}, x_{2}\right)^{T}$, and the initial data $u_{0}(x)=u_{1}(x)=\sin \left(\pi x_{1}\right)+\sin \left(2 \pi x_{1}\right)$. 
(a)

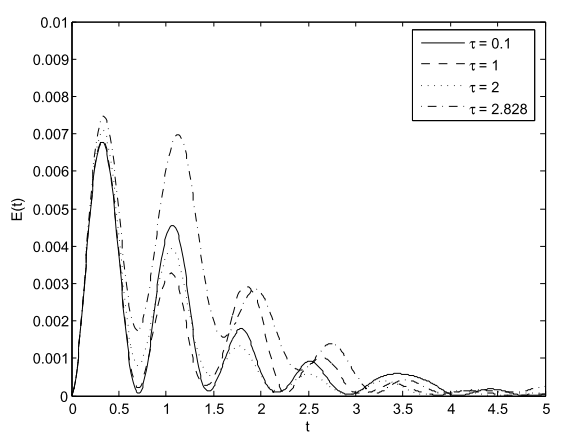

(c)

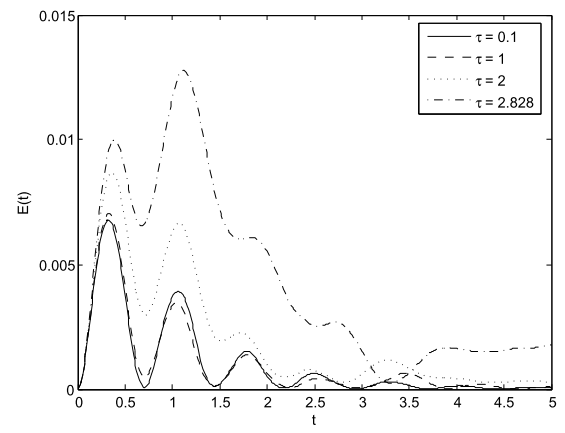

(b)

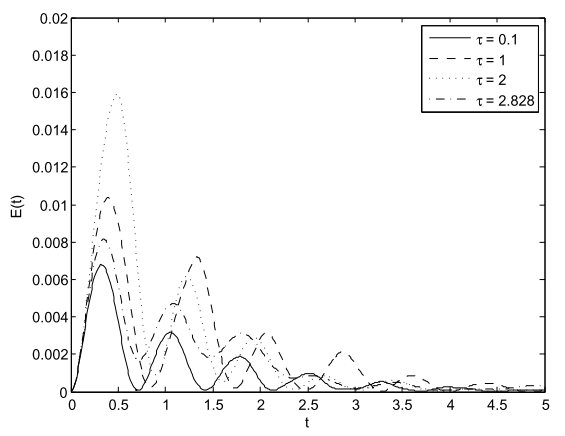

(d)

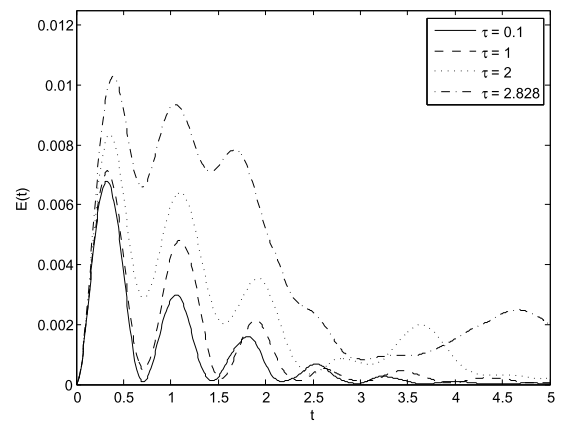

Figure 9 The energy $E_{0}(t)$ of the wave system (1.6)-(1.10) for different delays when $a(x)=e^{\left(x_{1} x_{2}\right)}$ with: a) $k=0.1$; b) $k=-0.1$; c) $k=0.2$; d) $k=-0.2$

First, we choose $a(x)=1$ and vary the values of $k$. It has been observed that the dynamics of the system (3.18)-(3.21) is exponentially stable if $k \geq 0$ and unstable for $k<0$. Figure 10 is a 2-d solution of $u(x, t)$ at it evolves in time for $k=1$, and Fig. 11a presents the corresponding energy $E(t)$ as defined by (3.17) versus time. It is clear that in this case, the dynamics of the system exponentially converges to the zero solution. Figure $11 \mathrm{~b}$ is a plot of the energy $E(t)$ for different positive values of $k$, indicating that the energy converges exponentially faster as the value of $k$ increases. On the other hand, Fig. 11c shows that the dynamics of the system is unstable when $k=-0.1$.

Then, we let $k=1$ and modify $a(x)$. Figure $11 \mathrm{~d}$ shows the convergence of the energy for various functions of $a(x)$ (i.e., $a(x)=1, a(x)=x_{1}$, and $a(x)=e^{x_{1}}$ ). The figure shows that faster convergence is achieved when $a(x)=e^{x_{1}}$, and this is due to the fact that $e^{x_{1}}>1>x_{1}$ for all $x_{1} \in[0,1]$.

\subsubsection{The 2-d wave system (1.16)-(1.20) with a boundary time-delay}

In this subsection, we go back to the 2-d wave equation (1.16)-(1.20) with a boundary delay $\tau$. The inial data are taken as follows: $u_{0}(x)=u_{1}(x)=\sin \left(\pi x_{1}\right)+\sin \left(2 \pi x_{1}\right)$, and $f(x, t)=$ $\sin \left(\pi x_{1}\right)$. The system is simulated for different parameters $k$, functions $a(x)$, and delays $\tau$. First, we choose the same value of $k=1$ and function $a(x)=1$ as in the case without delay presented in Sect. 3.2.1, but with delay $\tau=1$. Figure 12 presents the dynamics of $u(x, t)$ at different time, and Fig. 13 shows the corresponding energy $E(t)$ versus time. Figures 12 
(a)

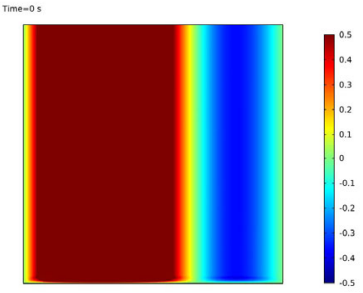

(d)

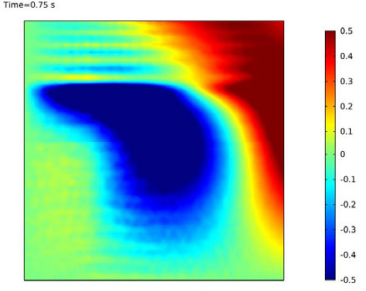

(g)

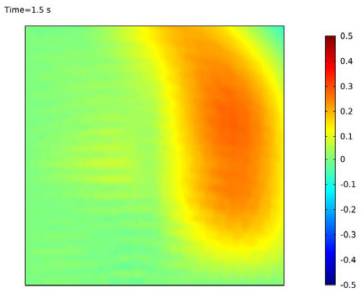

(b)

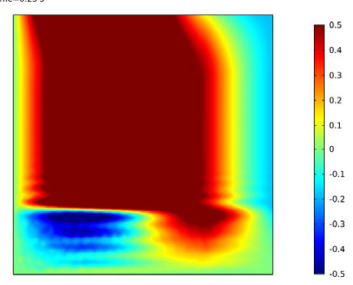

(e)

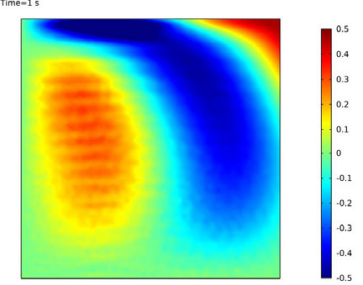

(h)

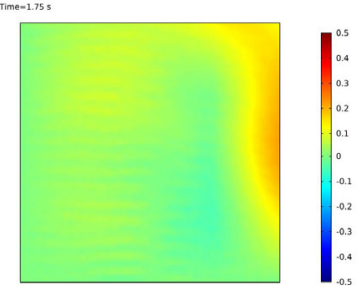

(c)

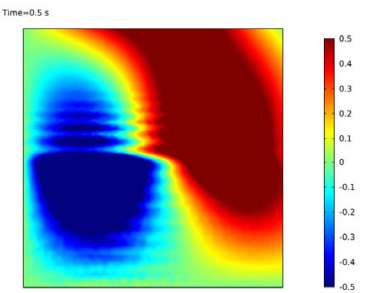

(f)

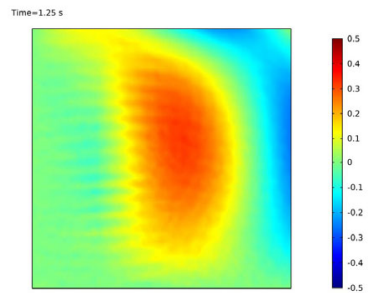

(i)

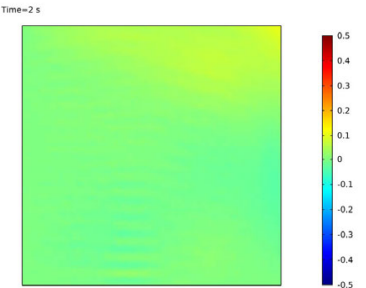

Figure 102 -d solutions of the wave equation without delay (3.18)-(3.21), when $a(x)=1$ and $k=1$, at different times: $t=0, \ldots, 2$

and 13 indicate that the presence of a delay destabilizes the dynamics of the system that was originally exponentially stable (compare with Figs. 10 and 11a). Moreover, choosing the function $a(x)=10$ and keeping the value of $k=1$ with a delay $\tau=1$ exponentially stabilize the dynamics of the 2-d wave equation to the zero solution (see Figs. 14 and 15a). It is worth mentioning that based on the above discussion, the choice of $a(x)$ plays an important role in the stability of the solution for the system (1.16)-(1.20) with a delay.

In turn, fixing $a(x)=1$ and a delay $\tau=1$ and choosing different values of $k$ change the dynamics of the 2-d wave equation (1.16)-(1.20) depending on the value of $k$. Figure 15b shows that the dynamics becomes more unstable as the value of $k$ increases from 0.1 to 0.4 . On the other hand, if the values of $k$ increase from -0.4 to -0.1 , the dynamics becomes more stable (see Fig. 15c). These observations indicate that if $a(x)=1$ and $|k|<0.3$, then the 2-d wave equation with a boundary delay $\tau=1$ is stable. However, this is not true if we change the function $a(x)=3$, where it is shown that the dynamics becomes more exponentially stable (see Fig. 15d). Figure 15e indicates similar observations to Fig. 7e when the delay is increased to $\tau=2$.

Now, to investigate the effect of the choice of the delay $\tau$ on the stability of the system (1.16)-(1.20), we fix $k$ and $a(x)$ and vary $\tau$. Figure $15 f$ shows that as we increase the value of the delay $\tau$ while fixing $k=-0.4$ and $a(x)=1$, the system is still unstable. However, the rate of divergence of the energy decreases as the delay $\tau$ increases. Similar observations 
(a)

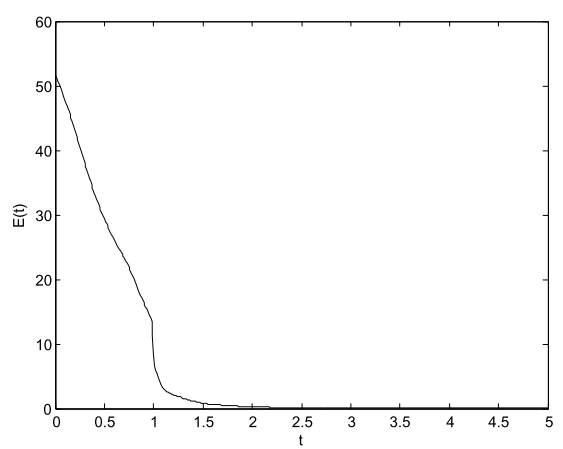

(c)

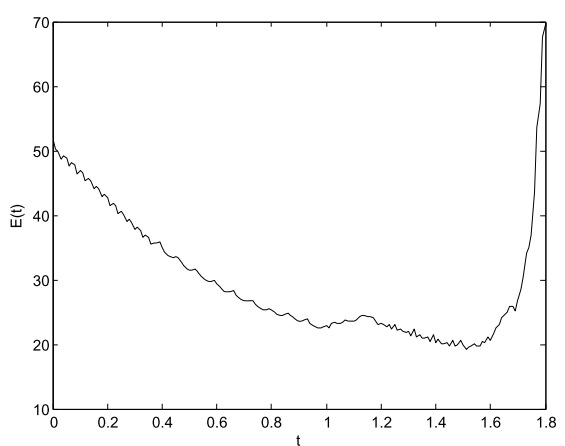

(b)

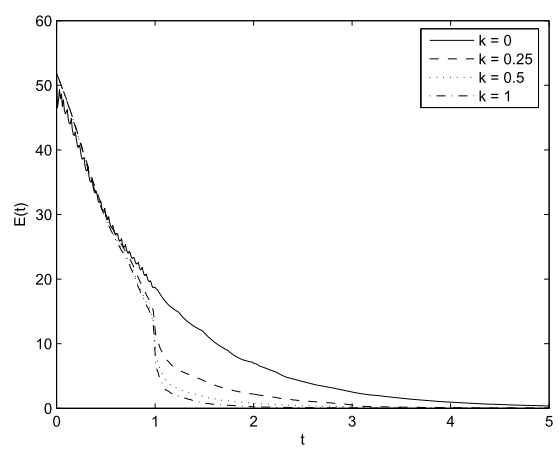

(d)

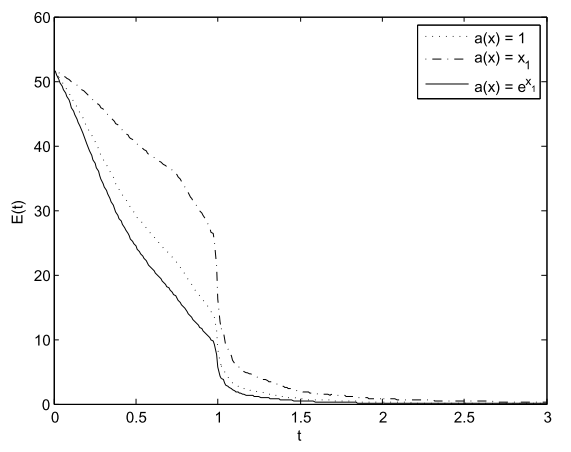

Figure 11 The energy $E(t)$ of the wave system without delay (3.18)-(3.21), when: a) $a(x)=1$ and $k=1$; b) $a(x)=1$, and for $k>0 ; c) a(x)=1$, and $k=-0.1 ; d) k=1$, and for various $a(x)$

are deduced for the cases $k=0.4$ and $a(x)=1$ (see Fig. 15g) and $k=1$ and $a(x)=1$ (see Fig. 15h).

Next, we investigate the stability of the system (1.16)-(1.20) for different values of $\tau$ when the support $\Omega_{1}$ of the function $a$ satisfies a geometric control condition (GCC) (i.e., $\left.\Omega_{1}=\left(\frac{1}{3}, \frac{2}{3}\right) x(0,1)\right)$. Furthermore, we fix $k$ and choose $a(x)=e^{\left(x_{1} x_{2}\right)}$, where $\left(x_{1}, x_{2}\right) \in \Omega_{1}$. Figures 16a-16d depict the energy, $E_{0}(t)$, versus time when $k=0.4,-0.4,1,-1$, respectively. Figure 16 indicates that the rate of divergence of the energy decreases as the time delay increases from $\tau=1$ to $\tau=4$.

\section{Appendix: The one-dimensional system (1.27)-(1.31)}

As mentioned in the introduction, the system (1.27)-(1.31) is exponentially stable in $L^{2}(0,1) \times H^{-1}(0,1)$ for any $k \in(-1,0)$. It suffices to proceed as in [5]. In fact, let $P=-\partial_{x}^{2}$ be the unbounded operator in $Y=H^{-1}(0,1)$ with domain $Y_{1}=\mathcal{D}(P)=H_{0}^{1}(0,1)$.

Then, let $Q \in \mathcal{L}\left(\mathbb{R} ; H_{-\frac{1}{2}}\right)$ such that

$$
Q r=P_{-1} L r, \quad \forall r \in \mathbb{R}, \quad Q^{*} s=\frac{\partial\left[\left(-\partial_{x}^{2}\right)^{-1} s\right]}{\partial x}(1), \quad \forall s \in \mathcal{D}\left(P^{\frac{1}{2}}\right)=Y_{\frac{1}{2}}=L^{2}(\Omega),
$$

where $Y_{-\frac{1}{2}}=\left(\mathcal{D}\left(P^{\frac{1}{2}}\right)\right)^{\prime}$ (the duality is in the sense of $Y$ ) and $P_{-1}$ is the extension of $P$ to $Y$, namely for all $h \in Y$ and $\varphi \in \mathcal{D}(P), P_{-1} h$ is the unique element in $Y_{-1}=(\mathcal{D}(P))^{\prime}$ (the duality 
(a)

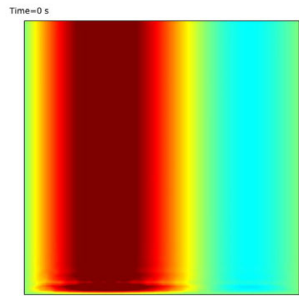

(d)

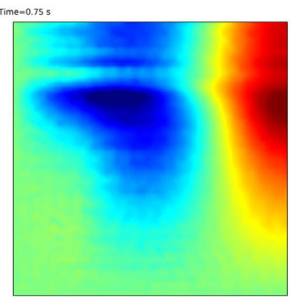

(g)

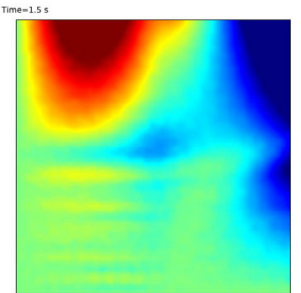

(b)
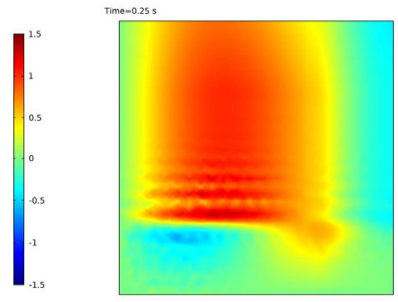

(e)
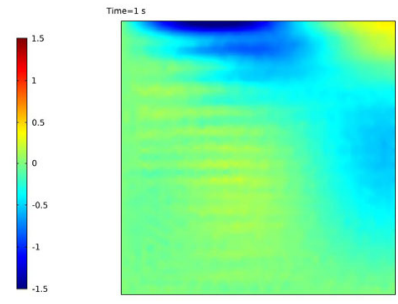

(h)
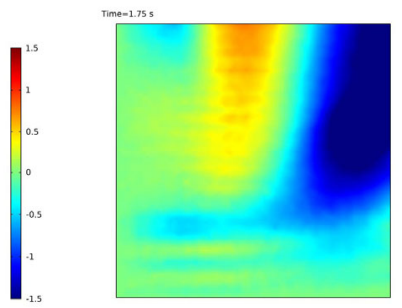
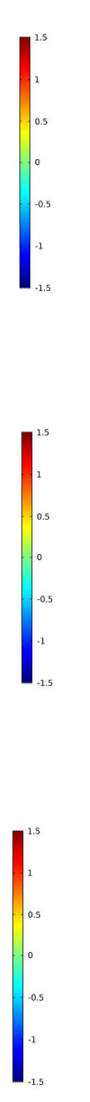

(c)

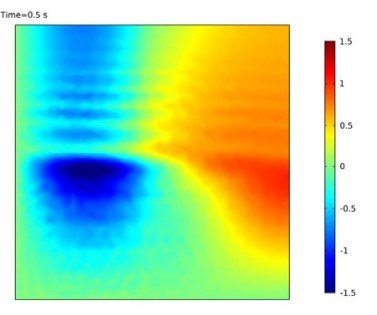

(f)

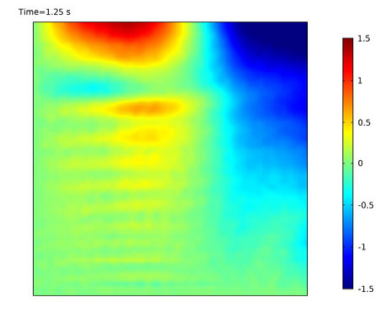

(i)

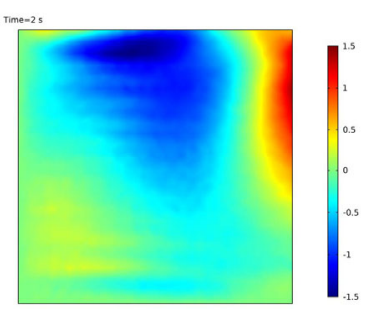

Figure 12 - $d$ solutions of the wave equation (1.16)-(1.20) with a delay $\tau=1$ when $a(x)=1$ and $k=1$, at different times: $t=0, \ldots, 2$

Figure 13 The energy $E(t)$ of the system (1.16)-(1.20) with a delay $\tau=1$ when $a(x)=1$, and $k=1$

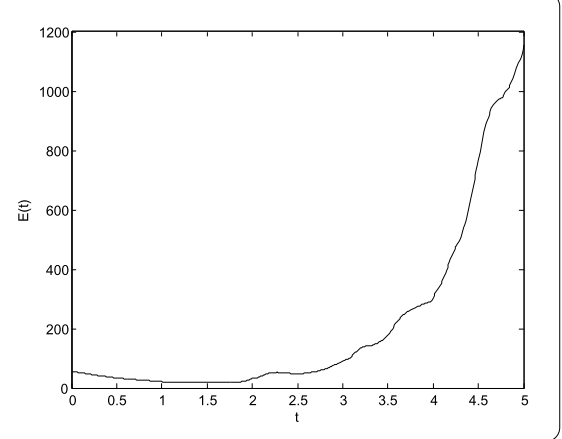

is in the sense of $Y$ ) such that

$$
\left\langle P_{-1} h, \varphi\right\rangle_{Y_{-1}, Y_{1}}=\langle h, P \varphi\rangle_{Y}
$$

Furthermore, $L \in \mathcal{L}\left(\mathbb{R} ; L^{2}(0,1)\right)$ is the Dirichlet mapping such as $\forall v \in \mathbb{R}, L v=g$ is the unique solution of

$$
\partial_{x}^{2} g=0, \quad g(0)=0, \quad g(1)=v
$$


(a)

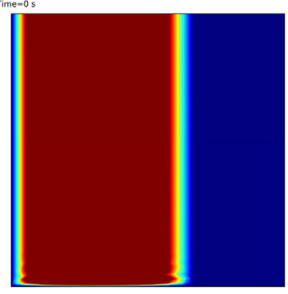

(d)

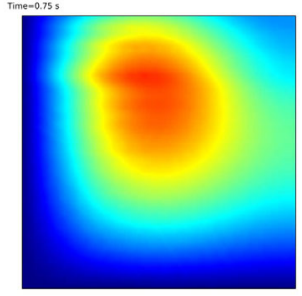

(g)

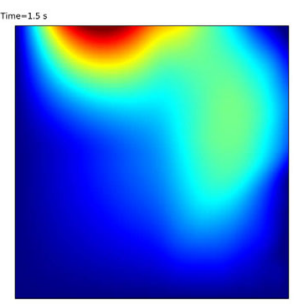

(b)

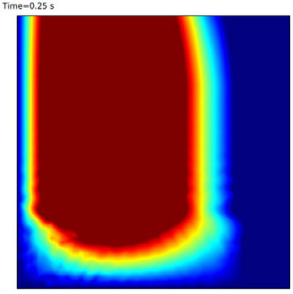

(e)

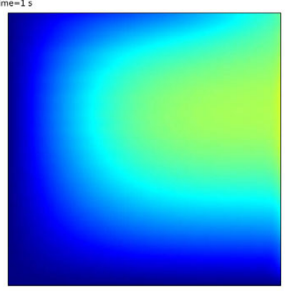

(h)

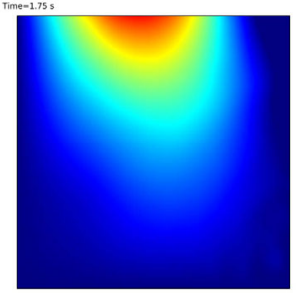

(c)

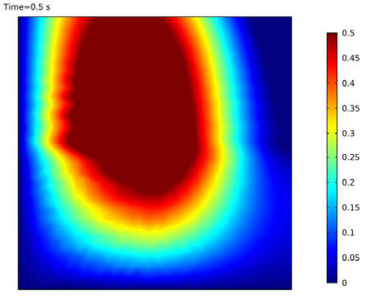

(f)

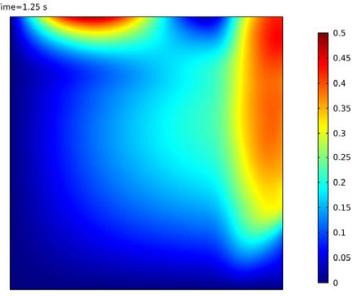

(i)

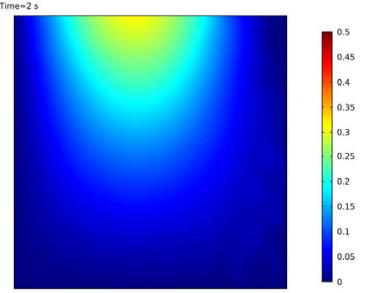

Figure 142 -d solutions of the wave equation (1.16)-(1.20) with a delay $\tau=1$ when $a(x)=10$ and $k=1$, at several times: $t=0, \ldots, 2$

Now, consider the Hilbert space $\mathcal{Y}:=Y_{\frac{1}{2}} \times Y$ and the unbounded linear operator:

$$
\mathcal{P}: \mathcal{D}(\mathcal{P})=Y_{1} \times Y_{\frac{1}{2}} \subset \mathcal{Y} \longrightarrow \mathcal{Y}, \quad \mathcal{P}(y, z)=(z,-P y), \forall(y, z) \in \mathcal{D}(\mathcal{P})
$$

The operator $(\mathcal{P}, \mathcal{D}(\mathcal{P}))$ defined by (4.1), generates a strongly continuous semigroup of contractions $(\mathcal{S}(t))_{t \geq 0}$ on $\mathcal{Y}$. On the other hand, $\left(\mathcal{S}_{-1}(t)\right)_{t \geq 0}$ denotes the extension of $(\mathcal{S}(t))_{t \geq 0}$ to $(\mathcal{D}(\mathcal{P}))^{\prime}:=Y \times Y_{-\frac{1}{2}}$, where $Y_{-\frac{1}{2}}=\left(\mathcal{D}\left(P^{\frac{1}{2}}\right)\right)^{\prime}$ and the duality is in the sense of $Y$.

We have the following result whose proof can be obtained similarly to [5]:

Theorem 4.1 The system (1.27)-(1.31) is well-posed in the following sense: for each $\left(u_{0}, u_{1}\right) \in \mathcal{Y}$, the solution of $(1.27)-(1.31)$ is given by

$$
\left(\begin{array}{c}
u(t) \\
u_{t}(t)
\end{array}\right)=\left\{\begin{array}{l}
\left(\begin{array}{c}
u^{0}(t) \\
u_{t}^{0}(t)
\end{array}\right)=\mathcal{S}(t)\left(\begin{array}{c}
u_{0} \\
u_{1}
\end{array}\right), \quad t \in[0,2], \\
\left(\begin{array}{c}
u^{j}(t) \\
u_{t}^{i}(t)
\end{array}\right)=\mathcal{S}(t-2 j)\left(\begin{array}{c}
w^{j-1}(2 j) \\
u_{t}^{j-1}(2 j)
\end{array}\right)-\int_{2 j}^{t} \mathcal{S}_{-1}(t-s)\left(\begin{array}{c}
0 \\
k Q^{*} u_{t}^{j-1}(s-2)
\end{array}\right) d s, \\
t \in[2 j, 2(j+1)], \quad j \geq 1,
\end{array}\right.
$$


(a)

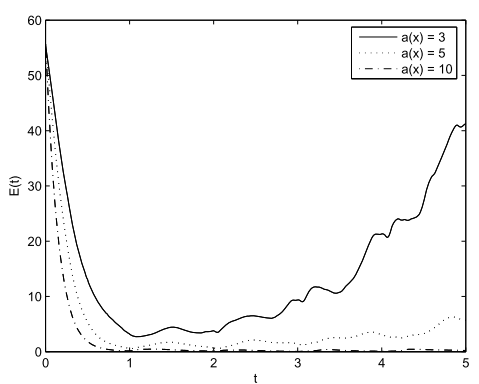

(c)

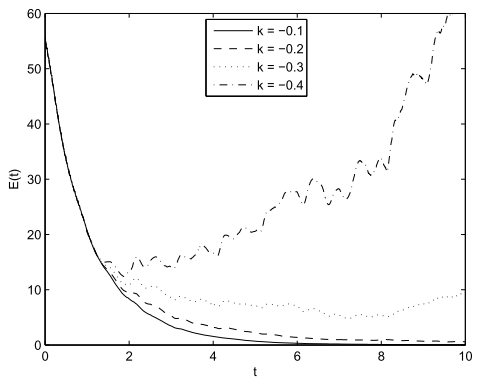

(e)

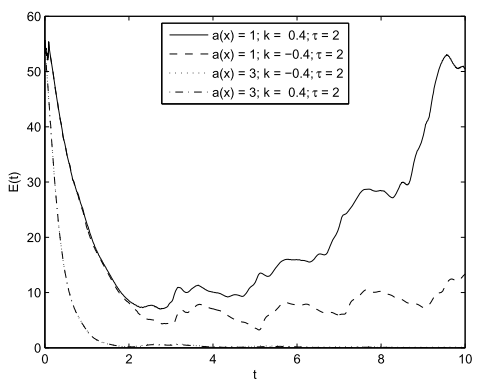

(g)

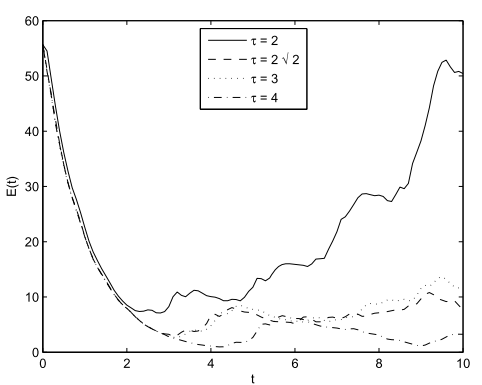

(b)

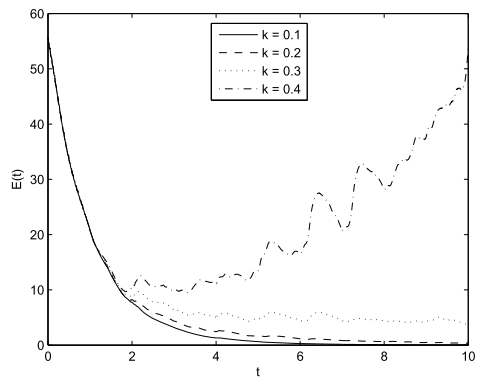

(d)

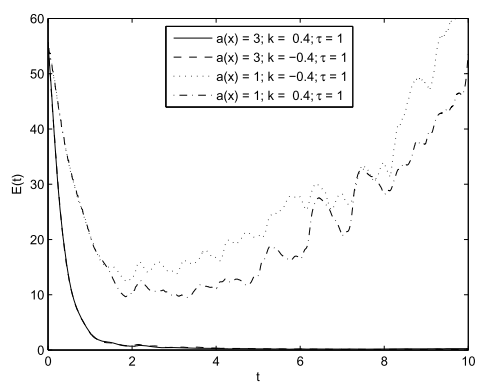

(f)

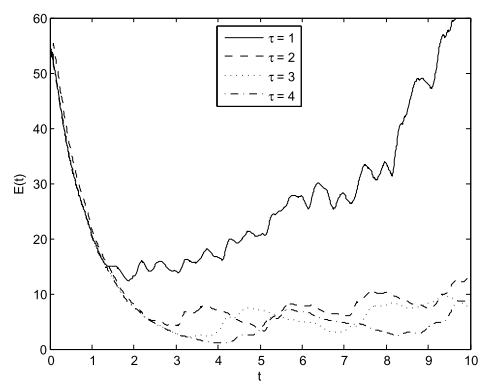

(h)

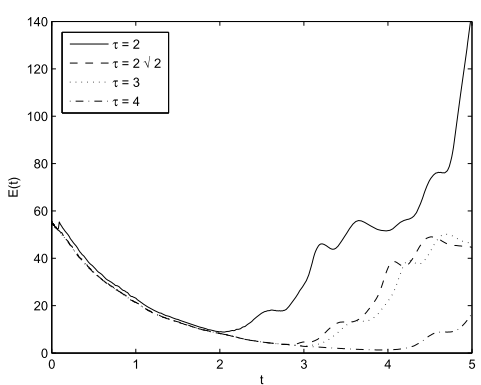

Figure 15 The energy $E(t)$ of the wave system (1.16)-(1.20) with: a) $\tau=1$ when $k=1$, and for different functions $a(x) ; b) \tau=1, k$ is positive and when $a(x)=1 ; c) \tau=1, k$ is negative and when $a(x)=1 ; d) \tau=1$, $a(x)=1$ and $a(x)=3$, and for numerous values of $k ; e) \tau=1, a(x)=1$ and $a(x)=3$, and for several values of $k ; f$ ) different delays when $a(x)=1$ and for $k=-0.4 ; g)$ various delays when $a(x)=1$ and for $k=0.4 ; h$ ) different delays when $a(x)=1$ and for $k=1$ 
(a)

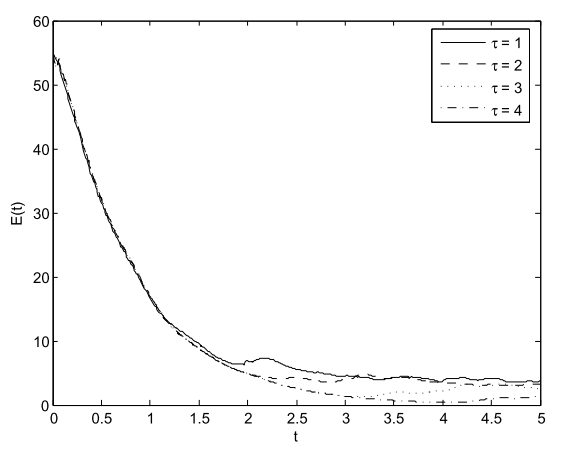

(c)

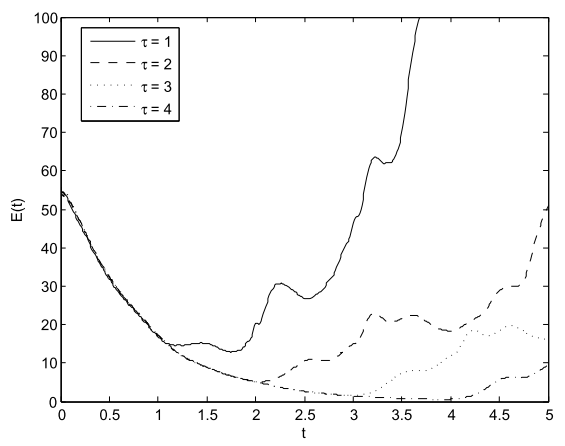

(b)

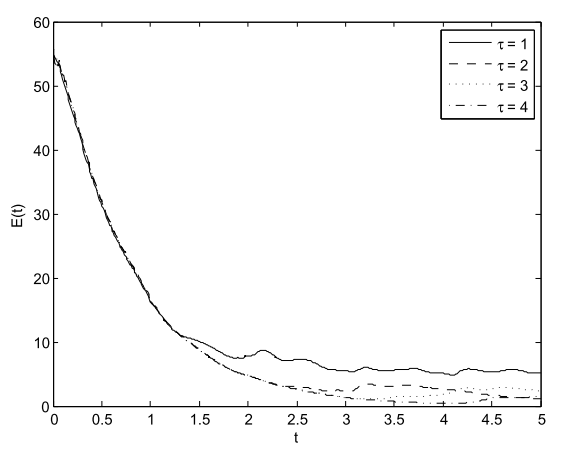

(d)

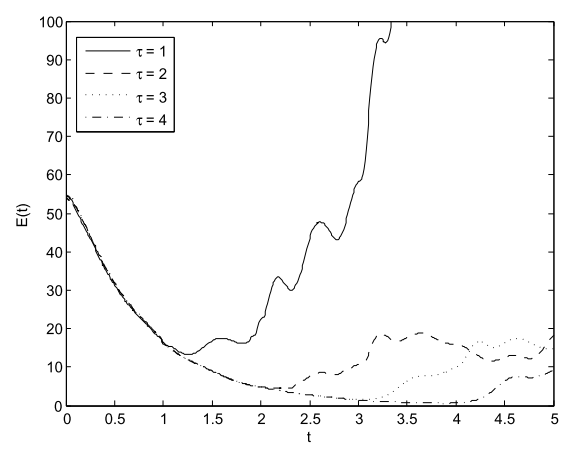

Figure 16 The energy $E(t)$ of the wave system (1.16)-(1.20) with $a(x)=e^{\left(x_{1} x_{2}\right)}$ and for different delays when: a) $k=0.4 ; b) k=-0.4$; c) $k=1$; d) $k=-1$

and satisfies $\left(u^{j}, u_{t}^{j}\right) \in C([2 j, 2(j+1)], \mathcal{Y}), j \in \mathbb{N}$.

With regard to the exponential stability of (1.27)-(1.31), we have:

Theorem 4.2 If $k \in(-1,0)$, then for all initial data in $\mathcal{Y}$, the solution of problem (1.27)(1.31) satisfies

$$
\left\|\left(u, u_{t}\right)\right\|_{L^{2}(0,1) \times H^{-1}(0,1)} \leq M e^{-\omega t}\left\|\left(u_{0}, u_{1}\right)\right\|_{L^{2}(0,1) \times H^{-1}(0,1)}, \quad \forall t \geq 0,
$$

where $M>0$ depends on the initial data and $k$, while $\omega>0$ depends on the feedback gain $k$.

Proof It suffices to seek a solution $u$ by invoking the D'Alembert formula

$$
u(x, t)=F(x+t)-F(t-x), \quad x \in(0,1), t \geq 0,
$$

where $F$ is a function to be found in $H_{\mathrm{loc}}^{1}(-1,+\infty)$ so that $u$ satisfies (1.27)-(1.31), and then use the same arguments as in [5] (see p. 5-7). 


\section{Acknowledgements}

The authors would like to thank the editor and the anonymous referee for their valuable corrections, suggestions, and comments that truly improved the quality of the manuscript.

Funding

Not Applicable

Availability of data and materials

The authors declare that all data and materials in this paper are available and veritable.

\section{Declarations}

\section{Competing interests}

The authors declare that they have no competing interests.

\section{Authors' contributions}

This is to declare that all authors have read and agreed to the published version of the manuscript.

\section{Author details}

${ }^{1}$ UR Analysis and Control of PDEs, UR13ES64, Department of Mathematics, Faculty of Sciences of Monastir, University of Monastir, 5019 Monastir, Tunisia. ${ }^{2}$ Department of Mathematics, Faculty of Science, Kuwait University, Safat 13060, Kuwait.

\section{Publisher's Note}

Springer Nature remains neutral with regard to jurisdictional claims in published maps and institutional affiliations.

Received: 9 May 2021 Accepted: 31 January 2022 Published online: 14 February 2022

\section{References}

1. Ait Ben Hassi, E.M., Ammari, K., Boulite, S., Maniar, L.: Feedback stabilization of a class of evolution equations with delay. J. Evol. Equ. 1, 103-121 (2009)

2. Ammari, K.: Dirichlet boundary stabilization of the wave equation. Asymptot. Anal. 30,117-130 (2002)

3. Ammari, K., Chentouf, B.: Asymptotic behavior of a delayed wave equation without displacement term. Z. Angew. Math. Phys. 68, Article ID ID13 (2017)

4. Ammari, K., Chentouf, B.: On the exponential and polynomial convergence for a delayed wave equation without displacement. Appl. Math. Lett. 86, 126-133 (2018)

5. Ammari, K., Chentouf, B., Smaoui, N.: Boundary stabilization of a one-dimensional wave equation by a switching time-delay: a theoretical and numerical study (2020) 2007.12599

6. Ammari, K., Nicaise, S.: Stabilization of Elastic Systems by Collocated Feedback. Lecture Notes in Mathematics, vol. 2124. Springer, Cham (2015)

7. Ammari, K., Nicaise, S., Pignotti, C.: Feedback boundary stabilization of wave equations with interior delay. Syst. Control Lett. 59, 623-628 (2010)

8. Ammari, K., Nicaise, S., Pignotti, C.: Stabilization by switching time-delay. Asymptot. Anal. 83, 263-283 (2013)

9. Bardos, C., Halpern, L., Lebeau, G., Rauch, J., Zuazua, E.: Stabilisation de l'équation des ondes au moyen d'un feedback portant sur la condition aux limites de Dirichlet. Asymptot. Anal. 4, 285-291 (1991)

10. Bardos, C., Lebeau, G., Rauch, J.: Sharp sufficient conditions for the observation, control and stabilization of waves from the boundary. SIAM J. Control Optim. 30, 1024-1065 (1992)

11. Baudouin, L., Crépeau, E., Valein, J.: Two approaches for the stabilization of the nonlinear KdV equation with boundary time-delay feedback. IEEE Trans. Autom. Control 64, 1403-1414 (2019)

12. Benaissa, A., Benguessoum, A., Messaoudi, S.A.: Energy decay of solutions for a wave equation with a constant weak delay and a weak internal feedback. Electron. J. Qual. Theory Differ. Equ. 2014, 11 (2014)

13. Bensoussan, A., Da Prato, G., Delfour, M.C., Mitter, S.K.: Representation and Control of Infinite-Dimensional Systems. Vol. 1, Systems \& Control: Foundations \& Applications. Birkhäuser, Boston (1992)

14. Burq, N.: Décroissance de l'énergie locale de l'équation des ondes pour le problème extérieur et absence de résonance au voisinage du réel. Acta Math. 180, 1-29 (1998)

15. Chen, G.: Energy decay estimates and exact boundary value controllability for the wave equation in a bounded domain. J. Math. Pures Appl. 58, 249-274 (1979)

16. Chen, G.: Control and stabilization for the wave equation in a bounded domain, Part I. SIAM J. Control Optim. 17, 66-81 (1979)

17. Chen, G.: Control and stabilization for the wave equation in a bounded domain, Part II. SIAM J. Control Optim. 19 114-122 (1981)

18. Chen, G.: A note on boundary stabilization of the wave equations. SIAM J. Control Optim. 19, 106-113 (1981)

19. Chung, J., Hulbert, G.M.: A time integration algorithm for structural dynamics with improved numerical dissipations: the generalized-alpha method. Appl. Mech. 60, 371-375 (1993)

20. Datko, R.: Not all feedback stabilized hyperbolic systems are robust with respect to small time delays in their feedbacks. SIAM J. Control Optim. 26, 697-713 (1988)

21. Datko, R., Lagnese, J., Polis, P.: An example on the effect of time delays in boundary feedback stabilization of wave equations. SIAM J. Control Optim. 24, 152-156 (1985)

22. Ferhat, Hakem, A.: Global existence and energy decay result for a weak viscoelastic wave equations with a dynamic boundary and nonlinear delay term. Comput. Math. Appl. 71, 779-804 (2016) 
23. Guo, B.Z., Xu, C.Z., Hammouri, H.: Output feedback stabilization of a one-dimensional wave equation with an arbitrary time-delay in boundary observation. ESAIM, Contrôle Optim. Calc. Var. 18, 22-35 (2012)

24. Guzman, P., Marx, S., Cerpa, E.: Stabilization of the linear Kuramoto-Sivashinsky equation with a delayed boundary control (2019). hal-02002453

25. Lagnese, J.: Decay of solutions of the wave equations in a bounded region with boundary dissipation. J. Differ. Equ. $50,163-182(1983)$

26. Lagnese, J.: Note on boundary stabilization of wave equations. SIAM J. Control Optim. 26, 1250-1256 (1988)

27. Lasiecka, l., Triggiani, R.: Uniform exponential decay in a bounded region with $L^{2}\left(0, \infty ; L^{2}(\Gamma)\right)$-feedback control in the Dirichlet boundary conditions. J. Differ. Equ. 66, 340-390 (1987)

28. Lebeau, G.: Equation des ondes amorties. In: Boutet de Monvel, A., et al. (eds.) Algebraic and Geometric Methods in Mathematical Physics. Math. Phys. Stud., vol. 19, pp. 73-109. Kluwer Academic, Dordrecht (1996)

29. Lions, J.L., Magenes, E.: Problèmes aux limites non homogènes et applications, vol. 1. Dunod, Paris (1968)

30. Liu, Z., Zheng, S.: Semigroups Associated with Dissipative Systems. Chapman \& Hall, Boca Raton (1999)

31. Nicaise, S., Pignotti, C.: Stability and instability results of the wave equation with a delay term in the boundary or internal feedbacks. SIAM J. Control Optim. 45, 1561-1585 (2006)

32. Nicaise, S., Pignotti, C.: Stabilization of the wave equation with boundary or internal distributed delay. Differ. Integral Equ. 21, 935-958 (2008)

33. Nicaise, S., Valein, J.: Stabilization of second order evolution equations with unbounded feedback with delay. ESAIM Control Optim. Calc. Var. 16, 420-456 (2010)

34. Tataru, D.: On the regularity of boundary traces for the wave equation. Ann. Sc. Norm. Super. Pisa, Cl. Sci. 26, 185-206 (1998)

35. Tucsnak, M., Weiss, G.: Observation and Control for Operator Semigroups. Birkhäuser, Basel (2009)

36. Zuazua, E.: Exponential decay for the semilinear wave equation with locally distributed damping. Commun. Partial Differ. Equ. 15, 205-235 (1990)

\section{Submit your manuscript to a SpringerOpen ${ }^{\circ}$ journal and benefit from:}

- Convenient online submission

- Rigorous peer review

- Open access: articles freely available online

- High visibility within the field

- Retaining the copyright to your article

Submit your next manuscript at $\gg$ springeropen.com 